\title{
Universal Correlations of Coulomb Blockade Conductance Peaks and the Rotation Scaling in Quantum Dots
}

\author{
Y. Alhassid and H. Attias \\ Center for Theoretical Physics, Sloane Physics Laboratory, Yale University, \\ New Haven, Connecticut 06520
}

(Submitted October 1995)

\begin{abstract}
We show that the parametric correlations of the conductance peak amplitudes of a chaotic or weakly disordered quantum dot in the Coulomb blockade regime become universal upon an appropriate scaling of the parameter. We compute the universal forms of this correlator for both cases of conserved and broken time reversal symmetry. For a symmetric dot the correlator is independent of the details in each lead such as the number of channels and their correlation. We derive a new scaling, which we call the rotation scaling, that can be computed directly from the dot's eigenfunction rotation rate or alternatively from the conductance peak heights, and therefore does not require knowledge of the spectrum of the dot. The relation of the rotation scaling to the level velocity scaling is discussed. The exact analytic form of the conductance peak correlator is derived at short distances. We also calculate the universal distributions of the average level width velocity for various values of the scaled parameter. The universality is illustrated in an Anderson model of a disordered dot.
\end{abstract}

PACS numbers: 73.40.Gk, 05.45.+b, 73.20.Dx, 72.20.My 


\section{INTRODUCTION}

A quantum dot [1] is an isolated 2-D region of a few $\mu \mathrm{m}$ or less in length to which $\mathcal{N}$ electrons (typically several hundreds) are confined by an electrostatic potential. The dots can be weakly coupled via tunnel barriers to external leads in order to study their transport properties. For sufficiently low temperatures the conductance of the dot exhibits equally spaced peaks with increasing gate voltage [2]. This phenomenon can be explained within a model [3] where the leads are assumed to behave like electron reservoirs in thermal equilibrium at temperature $T$ and chemical potential $E_{F}$, and the dot electrons are treated as a free Fermi gas with the Coulomb interaction taken into account only through charging effects. In this framework each successive peak corresponds to a single-electron tunneling event. For an additional electron to tunnel from the leads into the dot and occupy the ground state of the resulting $\mathcal{N}+1$-electron gas, it has to overcome the Coulomb repulsion energy of the dot electrons. $E_{F}$ must therefore increase from its value at the last tunneling event by $\Delta E_{F} \approx e^{2} / C+\Delta$ where $C$ is the capacitance between the dot and the reservoir and $\Delta=E_{\mathcal{N}+1}-E_{\mathcal{N}}$ is the spacing between the single-particle energies $E_{\mathcal{N}}$. This condition provides a set of values $\left\{E_{F}\right\}$ at which tunneling is possible, resulting in peaks in the conductance $G\left(E_{F}\right)$ of the dot. The suppression of tunneling between the peaks by Coulomb repulsion is called Coulomb blockade.

Since the charging energy $e^{2} / C \gg \Delta$, these peaks are equally spaced as a function of $E_{F}$ (for $k T<e^{2} / C$ ) and can be observed by increasing the gate voltage which controls $E_{F}$. The resulting oscillations in the measured conductance are known as Coulomb blockade oscillations. In the regime of isolated resonances $\bar{\Gamma}_{\lambda} \ll \Delta$ where $\Gamma_{\lambda}$ is the decay rate from the single-particle level $E_{\lambda}$ into the leads, and for temperatures $k T \ll \Delta$, the conductance is dominated by the contribution of the single level closest to $E_{F}$. In the temperature regime $k T \gg \bar{\Gamma}_{\lambda}$, the width of the peaks is $\sim k T$ independent of the individual decay rates.

The peak amplitudes are found to fluctuate strongly [2]. These fluctuations have been explained [4] by a statistical theory based on the assumption that the Hamiltonian of the dot can be described by random-matrix theory (RMT) [5, 6]. This assumption is valid either in the diffusive regime (for weak disorder) or when the classical dynamics of the electron inside the dot is chaotic [7] due to irregularities in the confining potential. The full probability distribution of the conductance was derived in this framework and found to change dramatically when time reversal symmetry is broken due to the presence of a magnetic field [4].

Recent experiments have been probing the dot's conductance as a function of the external magnetic field or a changing shape. Of particular interest are the correlations of the conductance peaks at different values of the external parameter (magnetic field, shape, etc.). For ballistic cavities (open dots), conductance correlations in a magnetic field were studied extensively, both theoretically for a large number of channels using the semiclassical approximation [8] and the supersymmetry method [9], and experimentally [10]. Recently these correlations were measured also as a function of the dot's shape [11]. Such open dots are characterized by many overlapping resonances and exhibit properties that are analogous to Ericson's fluctuations in nuclear reactions [12]. However, until recently, very little was known about parametric conductance correlations in the Coulomb blockade regime of isolated resonances, which is analogous to the neutron resonance regime in the compound 
nucleus [13,5].

In this paper we focus on the parametric correlation function of the conductance peaks

$$
c_{G}\left(x-x^{\prime}\right)=\overline{\tilde{G}(x) \tilde{G}\left(x^{\prime}\right)}, \quad \tilde{G}(x)=\frac{G(x)-\bar{G}}{\sqrt{\overline{\bar{G}^{2}}-\bar{G}^{2}}}
$$

where $x$ is any controllable parameter and $G(x)$ is the conductance peak amplitude for a given value $x$ of the parameter. In Ref. [14] we have proposed and derived for the first time the universal form of this correlator in the Coulomb blockade regime by using the framework of a Gaussian process (GP) [15, 16] that generalizes the Gaussian ensembles (GE) to situations where the chaotic or disordered Hamiltonian depends on a parameter. A particular realization of a GP has been introduced in Ref. [17] to study the statistics of avoided crossings in chaotic systems. Universal parametric correlation of spectra (e.g. level velocities) have been derived in Ref. [18, 19]. However, the calculation of the conductance correlations requires knowledge of statistical properties of the eigenfunctions. The universality of all

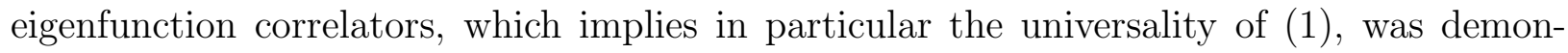
strated in [15]. Following our predictions, the conductance correlator has recently been measured for the situation of broken time-reversal symmetry where $x$ is a magnetic field [20] and was found to be in good agreement with theory [14]. The conductance distributions for one-channel symmetric leads were also measured [21,20] recently and are in accord with the theoretical distributions derived in Ref. [4].

For a dot with reflection symmetry we derive an even more remarkable result: the conductance correlator is independent of the details of the channels in each lead such as their number and degree of correlations, and it reduces to the resonance width correlator for single-channel leads. However, the conductance correlations are sensitive to the absence or presence of a magnetic field. To demonstrate the universality of our results we test them in an Anderson model of a disordered quantum dot.

Another problem that we address is the difficulty in performing the usual level velocity parameter scaling in experimental situations because of the inaccessibility of the spectrum, a Coulomb blockade effect. One can trace the variation of the ground state energy of $\mathcal{N}$ electrons in the dot as a function of the parameter by following the corresponding conductance peak. However it is not possible to measure the level spacing $\Delta$ which is needed for the level velocity scaling. Though $\Delta$ may be estimated theoretically, e.g. from free Fermi gas, its value may be modified by the electrons' interaction. We find a new scaling procedure, with a scaling factor which depends on the eigenfunctions alone and can be extracted from the level width or the conductance peak [14]. This should make it possible to extract the scaling directly from the conductance peaks data. The scaling factor is interpreted as the average rotation rate of the eigenvectors (with respect to $x$ ) or as the rms conductance velocity.

We remark that the conductance correlator in a symmetric dot is identical to the parametric correlator of the eigenfunction intensity at a fixed spatial point of a chaotic system, and therefore can also be measured in microwave cavity experiments [22,23].

The outline of the paper is as follows. In Section II we briefly review the theory of conductance in quantum dots in their Coulomb blockade regime using $R$-matrix formalism [24 26], or alternatively - resonance theory [26]. Both formalisms are particularly suitable for making the connection to RMT [4,22,28]. In Section III we derive and demonstrate the 
universal parametric correlations of the conductance peaks. We consider symmetric dots and show that in this case $c_{G}\left(x-x^{\prime}\right)$ is not only universal upon an appropriate scaling of the external parameter, but also independent of the channel information. We compute its universal form using a simple GP for both cases of conserved and broken time reversal symmetry. In section IV we introduce the new scaling procedure based on the eigenfunction rotation rate. The scaling factor diverges logarithmically for the orthogonal case but we describe a way to regularize it. Using perturbation theory, we derive analytically the width correlator at short distances and express the rotation scaling in terms of the RMS of the width velocity. We also derive a semiclassical expression which is system-dependent for the rotation scaling and compute it for the case when the parameter is an external magnetic field. This gives us the magnitude of the correlation field. In Section $\mathrm{V}$ we compute the universal distributions of the average width velocity for various values of the scaled $\Delta x$. In Section VI we discuss the universal parametric correlations of the conductance peaks in the general case of asymmetric dots with symmetric or asymmetric leads, and express the rotation scaling in terms of the RMS of the conductance velocity. Finally, our analytical results are verified in the completely solvable $N=2$ GP (Section VII).

\section{CONDUCTANCE IN QUANTUM DOTS}

\section{A. R-Matrix Formalism}

It is convenient to discuss the conductance of the dot in the framework of $R$-matrix theory, originally introduced for nuclear reactions that proceed through the compound nucleus formation and decay [25,26,24] and more recently applied to quantum dots [4,27,28]. We briefly review this formalism in the context of quantum dots but for the general case where there are several open channels at each lead. Let us consider a planar cavity-like dot in the $x-y$ plane with left and right leads of width $D_{l}, D_{r}$ attached to it at the lines of contact $x=x_{l}, x_{r} ; 0 \leq y \leq D_{l, r}$ which we denote by $\mathcal{C}$. The dot Hamiltonian has energies $E_{\lambda}$ whose corresponding eigenfunctions $\Psi_{\lambda}(\vec{r})$ vanish at the walls and satisfy an homogeneous boundary condition

$$
\partial \Psi_{\lambda} / \partial n-h_{l, r} \Psi_{\lambda}=0 \quad \text { for } \vec{r} \in \mathcal{C}
$$

where $\hat{n}$ is the normal to $\mathcal{C}$ (i.e. $\hat{n}= \pm \hat{x}$ ) and $h_{l, r}$ are constants.

We denote by $\phi_{c}^{i}$ a complete set of transverse wavefunctions, where $c$ is a channel index and $i=l, r$. For an open channel $\phi_{c}^{i}(y)=\sqrt{2 / D_{i}} \sin \left(\kappa_{c}^{i} y\right)$, where $\kappa_{c}^{i}=c \pi / D_{i}(c=$ $\left.1,2, \ldots, \Lambda_{i}\right)$ is the quantized transverse momentum. Inside each lead we can expand the scattering solution $\Phi$ at energy $E$ as

$$
\Phi(\vec{r})=\sum_{c=1}^{\Lambda_{i}} u_{c}^{i}(x) \phi_{c}^{i}(y) .
$$

The $R$-matrix, which relates the values of $\partial u_{c^{\prime}}^{i^{\prime}} / \partial n-h_{i^{\prime}} u_{c^{\prime}}^{i^{\prime}}$ to those of $u_{c}^{i}$ at $x=x_{i}$ can be expressed in terms of the resonances' eigenfunctions and energies through $R_{i^{\prime} c^{\prime} ; i c}(E)=$ $\sum_{\lambda} y_{c \lambda}^{i} y_{c^{\prime} \lambda}^{i^{\prime} *} /\left(E_{\lambda}-E\right)$ where 


$$
y_{c \lambda}^{i}=\sqrt{\frac{\hbar^{2}}{2 m}} \int_{\mathcal{C}} d l \phi_{c}^{i *} \Psi_{\lambda}
$$

is the reduced partial width amplitude for the decay from level $\lambda$ into channel $c$ in lead $i$.

If the resonances are isolated, i.e. their total width (see below) is much smaller than their average spacing $\Delta$, only one resonance whose energy $E_{\lambda}$ is closest to $E$ contributes to the $R$-matrix, and the cross section for scattering from channel $c$ in lead $i$ into channel $c^{\prime}$ in lead $i^{\prime}\left(i \neq i^{\prime}\right)$ is given by the Breit-Wigner resonance formula [24]

$$
\left|S_{i^{\prime} c^{\prime} ; i c}\right|^{2}=\frac{\Gamma_{c \lambda}^{i} \Gamma_{c^{\prime} \lambda}^{i^{\prime}}}{\left(E-E_{\lambda}\right)^{2}+\frac{1}{4}\left(\Gamma_{\lambda}^{l}+\Gamma_{\lambda}^{r}\right)^{2}} .
$$

Here $\Gamma_{c \lambda}^{i}$ and $\gamma_{c \lambda}^{i}$ are respectively the partial width and partial width amplitude of the resonance level $\lambda$ to decay into channel $c$ in lead $i$

$$
\Gamma_{c \lambda}^{i}=\left|\gamma_{\lambda}^{i}\right|^{2} ; \quad \gamma_{c \lambda}^{i}=\sqrt{2 k_{c}^{i} P_{c}^{i}} y_{c \lambda}^{i}
$$

where $k_{c}^{i}$ is the longitudinal channel momentum $\hbar^{2} k_{c}^{i 2} / 2 m+\hbar^{2} \kappa_{c}^{i 2} / 2 m=E$, and $P_{c}^{i}$ is the penetration factor to tunnel through the barrier in channel $c . \Gamma_{\lambda}^{i}=\sum_{c} \Gamma_{c \lambda}^{i}$ is the total decay width of level $\lambda$ into lead $i$.

The conductance of the dot at energy $E$ is given by Landauer's formula [29] $g(E)=$ $\left(e^{2} / h\right) \sum_{c c^{\prime}}\left|S_{r c^{\prime} ; l c}\right|^{2}$. At zero temperature (or for $\left.k T \ll \Gamma_{\lambda}\right)$ the conductance as a function of the Fermi energy is given by $g\left(E=E_{F}\right)$, while at finite temperature the conductance $g(E)$ due to one resonance level $\lambda$ has to be convoluted with the derivative of the Fermi function $f(\epsilon)=\left(1+e^{\epsilon / k T}\right)^{-1}$

$$
G\left(E_{F}\right)=-\int d E g(E) f^{\prime}\left(E-E_{F}\right) .
$$

In the temperature regime $\overline{\Gamma_{\lambda}} \ll k T \ll \Delta$ where Coulomb-blockade oscillations have been observed [2], the peak of $g(E)$ at $E=E_{\lambda}$ is much narrower than the peak of $-f^{\prime}\left(E-E_{F}\right)$ there and we can approximate (1) by

$$
G\left(E_{F}\right)=\frac{e^{2}}{h} \frac{\pi}{2 k T} \frac{\Gamma_{\lambda}^{l} \Gamma_{\lambda}^{r}}{\Gamma_{\lambda}^{l}+\Gamma_{\lambda}^{r}} \cosh ^{-2}\left(\frac{E_{F}-E_{\lambda}}{2 k T}\right) .
$$

The conductance has therefore a resonance shape as a function of $E_{F}$ centered at the resonance energy $E_{F}=E_{\lambda}$ with peak amplitude of

$$
G=\frac{e^{2}}{h} \frac{\pi}{2 k T} \frac{\Gamma_{\lambda}^{l} \Gamma_{\lambda}^{r}}{\Gamma_{\lambda}^{l}+\Gamma_{\lambda}^{r}}
$$

and width of $k T$. This formula has been derived in [3] using a different method.

We remark that a different modelling of a quantum dot assumes point-like contacts where each lead is composed of several such point contacts. One arrives at the same expression (9) for the conductance [30, except that each point contact $\mathbf{r}_{c}$ is considered as one channel and the corresponding reduced partial width is 31] $\gamma_{c \lambda}=\sqrt{\alpha_{c} \mathcal{A} \Delta / \pi} \Psi_{\lambda}\left(\mathbf{r}_{c}\right)$, where $\mathcal{A}$ is the area of the dot, $\Delta$ is the mean level spacing and $\alpha_{c}$ is a coupling parameter of the dot to the lead that represents a tunneling probability through the barrier. 


\section{B. Resonance Theory}

Eqs. (6) and (田), which relate the partial widths to the resonance eigenfunction in the dot, play a major role in the statistical theory for the conductance peaks (9), and it is therefore instructive to derive them directly from resonance theory [26] without using the $R$-matrix formalism.

A decaying state is described by the condition that the wavefunction has only an outgoing component in the external region (instead of the boundary condition (2) used in the $R$-matrix formalism). In our case a general solution $\Phi$ at energy $E$ has inside the leads the form (3) with $u_{c}(x)=A_{c} u_{c}^{+}(x)+B_{c} u_{c}^{-}(x)$ where $u_{c}^{ \pm}(x)$ are incoming and outgoing longitudinal waves propagating through a potential barrier $V_{c}(x)$ in channel $c$. The conditions $B_{c}=0$ in all channels determine a discrete set of complex energies $E_{\lambda}^{R}=E_{\lambda}+\delta E_{\lambda}-i \Gamma_{\lambda} / 2$, where $\delta E_{\lambda}$ is a real shift in the resonance energy and $\Gamma_{\lambda}=\sum_{c} \Gamma_{c \lambda}$ is the total resonance width. An equivalent boundary condition for determining the complex resonance energies is that the logarithmic derivative at the interface of the leads with the dot is equal to the logarithmic derivative of the outgoing wave

$$
\left.\frac{\partial}{\partial n} \ln u_{c}^{i}\right|_{x=x_{i}}=\left.h_{i c}^{+} \equiv \frac{\partial}{\partial n} \ln u_{c}^{i+}\right|_{x=x_{i}} .
$$

$h_{i c}^{+}$are generally complex, e.g. $h_{i c}^{+}= \pm i k_{c}^{i}$ in the absence of barrier (when $u_{c}^{+}$is a plane wave propagating in a direction away from the dot). The modification of the boundary conditions from (2) (where $h_{i}$ are real) to (10) allows the wavefunction to leak into the leads. The partial decay rate $\Gamma_{c \lambda} / \hbar$ into channel $c$ of lead $i$ is given by the current through the cross-section of the lead

$$
\frac{\Gamma_{c \lambda}^{i}}{\hbar}=\frac{\hbar}{2 i m} \int_{\mathcal{C}} d l\left(\Psi_{\lambda}^{*} \frac{\partial \Psi_{\lambda}}{\partial n}-\Psi_{\lambda} \frac{\partial \Psi_{\lambda}^{*}}{\partial n}\right)_{c}=\frac{\hbar}{2 i m}\left[u_{c}^{*} \frac{d u_{c}}{d n}-u_{c} \frac{d u_{c}^{*}}{d n}\right]_{x=x_{i}} .
$$

Using the boundary conditions (10) we find

$$
\Gamma_{c \lambda}^{i}=\frac{\hbar^{2}}{m} \operatorname{Im} h_{i c}^{+}\left|u_{c}\left(x_{i}\right)\right|^{2}
$$

which is exactly Eqs. (6) and (4) provided the penetration factor $P_{c}$ is defined by

$$
k_{c}^{i} P_{c}^{i} \equiv \operatorname{Im} h_{i c}^{+} .
$$

In the absence of a barrier $P_{c}^{i}=1$, and we expect $P_{c}^{i} \ll 1$ in the presence of a barrier.

\section{Statistical Model for Dots with Multi-channel Leads}

Eq. (9) indicates that the irregular fluctuations of the conductance peaks arise from fluctuations of the level widths $\Gamma_{c \lambda}^{i}$. We assume that the channel penetration factors $P_{c}^{i}$ have negligible dependence on the parameter, and in any case they vary smoothly with $x$. These fluctuations must then come from the eigenfunctions $\Psi_{\lambda}$ inside the dot, and have recently 
been accounted for by a statistical theory [4] based on the assumption that the Hamiltonian of the dot can be described by a $N \times N$ random matrix $H$ taken from the appropriate GE [5]. The dot's eigenfunctions $\Psi_{\lambda}$ are expanded in a complete set of solutions $\rho_{\mu}^{\lambda}$ inside the dot with the given energy $E_{\lambda}, \Psi_{\lambda}(\vec{r})=\sum_{\mu=1}^{N} \psi_{\mu}^{\lambda} \rho_{\mu}^{\lambda}(\vec{r})$, and the expansion coefficients $\psi_{\mu}^{\lambda}$ are assumed to constitute the components of the $\lambda$-th eigenvector of the random matrix $H$. The partial width amplitude to decay into channel $c$ (4) can then be expressed as a scalar product

$$
\gamma_{c \lambda}^{i}=\sum_{\mu=1}^{N} \phi_{c \mu}^{i *} \psi_{\mu}^{\lambda} \equiv\left\langle\phi_{c}^{i} \mid \psi_{\lambda}\right\rangle
$$

where $\phi_{c}^{i}$ is the channel vector defined by $\phi_{c \mu}^{i}=\sqrt{\hbar^{2} k_{c}^{i} P_{c}^{i} / m} \int_{\mathcal{C}} d l \phi_{c}^{i *} \rho_{\mu}$. In the point contact model, a similar formula (14) applies but with $\phi_{c \mu} \equiv \sqrt{\alpha_{c} \mathcal{A} \Delta / \pi} \rho_{\mu}^{*}\left(\mathbf{r}_{c}\right)$. Thus in the corresponding $N$-dimensional space, the partial width amplitude of a level is simply the projection of its corresponding eigenstate $\psi_{\lambda}$ on the channel vector $\phi_{c}$. We remark that the scalar product in Eq. (14) (that will be used throughout this paper) is different from the original scalar product defined in the spatial region occupied by the dot.

We define the channel correlation matrix $M$ of lead $i$ by $M_{c c^{\prime}}^{i}=\overline{\gamma_{c}^{i *} \gamma_{c^{\prime}}^{i}}$. Using the scalar product expression (14) for the partial width amplitude and the GE average $\overline{\psi_{\mu}^{\lambda} \psi_{\mu^{\prime}}^{\lambda}}=\frac{1}{N} \delta_{\mu \mu^{\prime}}$, we can rewrite $M$ in terms of the scalar product of the corresponding channels

$$
M_{c c^{\prime}}^{i}=\frac{1}{N}\left\langle\phi_{c}^{i} \mid \phi_{c^{\prime}}^{i}\right\rangle
$$

The norm of a channel vector is determined by the corresponding mean partial width through $\left\langle\phi_{c}^{i} \mid \underline{\phi_{c}^{i}}\right\rangle=N \overline{\Gamma_{c}^{i}}$. Channels $c, c^{\prime}$ are called uncorrelated if $\overline{\gamma_{c}^{i *} \gamma_{c^{\prime}}^{i}}=0$ and equivalent if $\overline{\Gamma_{c}^{i}}=\overline{\Gamma_{c^{\prime}}^{i}}$. In general channels can be correlated or non-equivalent and the channel vectors $\phi_{c}$ can therefore be non-orthogonal and have different norms. Channels in different leads are assumed to be uncorrelated. The matrices $M_{c c^{\prime}}^{l, r}$ thus contain all the channel information relevant for the statistical description of the dot's conductance through Eqs. (9), (6) and (14).

\section{CONDUCTANCE CORRELATIONS IN SYMMETRIC DOTS}

In experiments on quantum dots one can vary an external parameter $x$ such as the shape of the dot or the strength of an applied magnetic field and trace the corresponding change in the amplitude of a given conductance peak. We are then interested in calculating the conductance peak correlator (国), assuming the electron's dynamics inside the dot is chaotic (or disordered) at all values of $x$. In this section we show that the conductance correlator is universal upon an appropriate scaling of the parameter $x$ and we obtain its universal form. 


\section{A. Gaussian Random-Matrix Process}

To calculate the correlation between conductance peaks that belong to different parameter values it is necessary to use a random-matrix model which incorporates a parametric dependence. Such a model, the Gaussian random-matrix process (GP) which constitutes a natural generalization of Dyson's Gaussian ensemble (GE) [32] has recently been proposed and applied to the calculation of universal parametric correlation functions in chaotic and disordered systems 15.

A GP is a set of $N \times N$ random matrices $H(x)$ whose elements satisfy

$$
\begin{aligned}
\overline{H_{\lambda \sigma}(x)} & =0, \\
\overline{H_{\lambda \sigma}(x) H_{\mu \nu}\left(x^{\prime}\right)} & =\frac{a^{2}}{2 \beta} f\left(x-x^{\prime}\right) g_{\lambda \sigma, \mu \nu}^{(\beta)},
\end{aligned}
$$

where $g_{\lambda \sigma, \mu \nu}^{(\beta=1)}=\delta_{\lambda \mu} \delta_{\sigma \nu}+\delta_{\lambda \nu} \delta_{\sigma \mu}$ and $g_{\lambda \sigma, \mu \nu}^{(\beta=2)}=2 \delta_{\lambda \nu} \delta_{\sigma \mu}$. For situations with time-reversal invariance we have $\beta=1$ and $H(x)$ are real symmetric matrices, whereas for broken time-reversal symmetry $\beta=2$ and $H(x)$ are complex Hermitian. The corresponding GPs are termed Gaussian orthogonal process (GOP) and Gaussian unitary process (GUP), respectively.

A GP is characterized by the short distance behavior of its correlation function $f$

$$
f\left(x-x^{\prime}\right) \approx 1-\kappa\left|x-x^{\prime}\right|^{\eta},
$$

where $\kappa$ and $\eta(0<\eta \leq 2)$ are constants. The GP unfolded energies $\varepsilon_{\lambda}=E_{\lambda} / \Delta$ (where $\Delta$ is the average level spacing) satisfy a diffusion law [15, [16] at short distances

$$
\overline{\left(\Delta \varepsilon_{\lambda}\right)^{2}}=D|\Delta x|^{\eta}+\mathcal{O}\left(|\Delta x|^{2 \eta}\right)
$$

where

$$
D=\lim _{\Delta x \rightarrow 0} \frac{\overline{\left(\Delta \varepsilon_{\lambda}\right)^{2}}}{|\Delta x|^{\eta}}=\frac{4 N \kappa}{\pi^{2} \beta}
$$

plays the role of the diffusion constant. Upon the parameter scaling

$$
x \rightarrow \bar{x}=D^{1 / \eta} x
$$

the correlation function $f\left(x-x^{\prime}\right)$ (17) becomes $f \approx 1-\frac{\pi^{2} \beta}{4 N}\left|\bar{x}-\bar{x}^{\prime}\right|^{\eta}$, and all correlators become universal functions of $\left|\bar{x}-\bar{x}^{\prime}\right|^{\eta}$. This universality can also be demonstrated through Dyson's Brownian motion model [33]. The $\eta=2$ GPs are the only class of processes that are continuously differentiable in the parameter and therefore the ones suitable to describe most physical systems [16]. For $\eta=2$ the diffusion scaling (20) reduces to the level velocity scaling $D=C(0) \equiv \overline{\left(\partial \varepsilon_{\lambda} / \partial x\right)^{2}}$, first introduced in [18]. 


\section{B. The Conductance Parametric Correlator}

When the dot is symmetric under reflection around the $y$-axis we have $\Psi_{\lambda}(-x, y)=$ $\pm \Psi_{\lambda}(x, y)$, thus $\Gamma_{c \lambda}^{l}=\Gamma_{c \lambda}^{r} \equiv \Gamma_{c \lambda}$ and the peak conductance (9) becomes

$$
G=\frac{e^{2}}{h} \frac{\pi}{4 k T} \Gamma_{\lambda} .
$$

We take the dot Hamiltonian $H(x)$ to be a member of the appropriate GP with energies $E_{\lambda}(x)$ and eigenfunctions $\psi_{\lambda}(x)$. The conductance correlator (1) reduces in this case to the level width correlator

$$
c_{G}\left(x-x^{\prime}\right)=c_{\Gamma}\left(x-x^{\prime}\right)=\overline{\tilde{\Gamma}(x) \tilde{\Gamma}\left(x^{\prime}\right)}, \quad \tilde{\Gamma}(x)=\frac{\Gamma(x)-\bar{\Gamma}}{\sqrt{\bar{\Gamma}^{2}-\bar{\Gamma}^{2}}}
$$

where $\Gamma(x)=\sum_{c=1}^{\Lambda}\left|\left\langle\phi_{c} \mid \psi(x)\right\rangle\right|^{2}$ (see (14)). Here and in the following we omit the eigenvector label $\lambda$ and the lead label $i$. It is clear from the discussion in Section III.A that this correlator becomes universal under the diffusion scaling (20), for any given set of channel vectors $\phi_{c}$. Furthermore, due to the invariance of the GOP (GUP) under an $x$-independent orthogonal (unitary) transformation, this correlator can depend only on the correlation matrix $M$ in (15). However, we will prove below the stronger result that the width correlator is also independent of $M$.

Since $M$ is Hermitian and positive-definite, it can be diagonalized by a unitary transformation $U$ under which the channel vectors $\phi_{c}$ transform into an orthogonal set of eigenchannels $\bar{\phi}_{c}\left(\phi_{c}=\sum_{c^{\prime}} \bar{\phi}_{c^{\prime}} U_{c^{\prime} c}\right)$ and

$$
\bar{M}_{c c^{\prime}}=\frac{1}{N}\left\langle\bar{\phi}_{c} \mid \bar{\phi}_{c^{\prime}}\right\rangle=w_{c}^{2} \delta_{c c^{\prime}}
$$

where $w_{c}^{2}=\left\langle\bar{\phi}_{c} \mid \bar{\phi}_{c}\right\rangle / N$ are the (positive) eigenvalues of $M$. Since the total width (in each lead) is invariant under a unitary transformation, we have $\Gamma=\sum_{c}\left|\gamma_{c}\right|^{2}=\sum_{c}\left|\bar{\gamma}_{c}\right|^{2}$ (where $\bar{\gamma}_{c}=\left\langle\bar{\phi}_{c} \mid \psi_{\lambda}\right\rangle$ are the partial widths to decay to the eigenchannels) and the width correlator thus depends only on the eigenvalues $w_{c}^{2}$ of the correlation matrix. Defining the normalized eigenchannels $\left|\hat{\phi}_{c}\right\rangle=\left(\sqrt{N} w_{c}\right)^{-1}\left|\bar{\phi}_{c}\right\rangle$ we can express the width in terms of the partial widths to decay into an orthonormal set of channels

$$
\Gamma(x)=N \sum_{c=1}^{\Lambda} w_{c}^{2} \hat{\Gamma}_{c}(x), \quad \hat{\Gamma}_{c}(x) \equiv\left|\left\langle\hat{\phi}_{c} \mid \psi(x)\right\rangle\right|^{2},
$$

and the numerator of the width correlator (22) becomes

$$
\overline{\Gamma(x) \Gamma\left(x^{\prime}\right)}-\bar{\Gamma}^{2}=\sum_{c} w_{c}^{4}\left(\overline{\hat{\Gamma}_{c}(x) \hat{\Gamma}_{c}\left(x^{\prime}\right)}-{\overline{\hat{\Gamma}_{c}}}^{2}\right)+\sum_{c \neq c^{\prime}} w_{c}^{2} w_{c^{\prime}}^{2}\left(\overline{\hat{\Gamma}_{c}(x) \hat{\Gamma}_{c^{\prime}}\left(x^{\prime}\right)}-\overline{\hat{\Gamma}_{c}} \overline{\hat{\Gamma}_{c^{\prime}}}\right) .
$$

We now observe that the averaged quantities in (25) do not depend on the subscripts $c, c^{\prime}$ but only on the orthogonality relation $\left\langle\hat{\phi}_{c} \mid \hat{\phi}_{c^{\prime}}\right\rangle=0$. Indeed, if we chose a different pair 
$\hat{\varphi}_{c}, \hat{\varphi}_{c^{\prime}}$ satisfying $\left\langle\hat{\varphi}_{c} \mid \hat{\varphi}_{c^{\prime}}\right\rangle=0$ there would exist a unitary transformation $U$ such that $\left|\hat{\varphi}_{c}\right\rangle=U\left|\hat{\phi}_{c}\right\rangle$ and $\left|\hat{\varphi}_{c^{\prime}}\right\rangle=U\left|\hat{\phi}_{c^{\prime}}\right\rangle$ which could be used to rotate $H(x)$ at each $x$ into $U H(x) U^{\dagger}$ with eigenfunctions $U\left|\psi_{\lambda}(x)\right\rangle$, leaving $\hat{\Gamma}_{c}(x), \hat{\Gamma}_{c^{\prime}}\left(x^{\prime}\right)$ unchanged. Since the GP probability measure $P[H(x)] D[H(x)]$ (see [16]) is invariant under global rotation, the corresponding averages remain unchanged as well. We then rewrite (25) as

$\overline{\Gamma(x) \Gamma\left(x^{\prime}\right)}-\bar{\Gamma}^{2}=\left(\sum_{c} w_{c}^{4}\right)\left[\overline{\hat{\Gamma}_{1}(x) \hat{\Gamma}_{1}\left(x^{\prime}\right)}-{\overline{\hat{\Gamma}_{1}}}^{2}\right]+\left(\sum_{c \neq c^{\prime}} w_{c}^{2} w_{c^{\prime}}^{2}\right)\left[\overline{\hat{\Gamma}_{1}(x) \hat{\Gamma}_{2}\left(x^{\prime}\right)}-\overline{\hat{\Gamma}_{1}} \overline{\hat{\Gamma}_{2}}\right]$,

where the subscripts 1,2 refer to any orthonormal pair $\hat{\phi}_{c}, \hat{\phi}_{c^{\prime}}\left(c \neq c^{\prime}\right)$. Now the cross-channel correlator (second correlator on the r.h.s. of (26)) is related to the autochannel correlator (first term there) by

$$
\overline{\hat{\Gamma}_{1}(x) \hat{\Gamma}_{2}\left(x^{\prime}\right)}-\overline{\hat{\Gamma}_{1}} \overline{\hat{\Gamma}_{2}}=-\frac{1}{N-1}\left[\overline{\hat{\Gamma}_{1}(x) \hat{\Gamma}_{1}\left(x^{\prime}\right)}-{\overline{\hat{\Gamma}_{1}}}^{2}\right] .
$$

To prove (27) we use a complete basis of $N$ orthonormal channels $\hat{\phi}_{i} ; i=1, \ldots, N$. Multiplying the two normalization conditions $\sum_{i=1}^{N}\left|\hat{\gamma}_{i}(x)\right|^{2}=1$ and $\sum_{j=1}^{N}\left|\hat{\gamma}_{j}\left(x^{\prime}\right)\right|^{2}=1$ and averaging, we get

$$
N \overline{\left|\hat{\gamma}_{1}(x)\right|^{2}\left|\hat{\gamma}_{1}\left(x^{\prime}\right)\right|^{2}}+N(N-1) \overline{\left|\hat{\gamma}_{1}(x)\right|^{2}\left|\hat{\gamma}_{2}\left(x^{\prime}\right)\right|^{2}}=1
$$

Relation (28) leads immediately to (27). According to (27) the cross-channel correlator is negligible in the limit $N \rightarrow \infty$ compared with the autochannel correlator. From (26) we obtain

$$
\overline{\Gamma(x) \Gamma\left(x^{\prime}\right)}-\bar{\Gamma}^{2}=\left(\sum_{c} w_{c}^{4}-\frac{1}{N-1} \sum_{c \neq c^{\prime}} w_{c}^{2} w_{c^{\prime}}^{2}\right)\left[\overline{\hat{\Gamma}_{1}(x) \hat{\Gamma}_{1}\left(x^{\prime}\right)}-{\overline{\hat{\Gamma}_{1}}}^{2}\right],
$$

and therefore

$$
c_{\Gamma}\left(x-x^{\prime}\right)=\overline{\tilde{\hat{\Gamma}}_{1}(x) \tilde{\hat{\Gamma}}_{1}\left(x^{\prime}\right)} .
$$

This result (30) is remarkable in that the conductance correlator in a symmetric dot is not only universal but also independent of the number of channels in the leads, their associated mean decay widths and the correlations among them; it is given by the correlator of a dot with single-channel leads. The universal form of this function can be computed using any GP [15]. We choose the simple process [34]

$$
H(x)=H_{1} \cos x+H_{2} \sin x
$$

which is an $\eta=2 \mathrm{GP}$ with a correlation function $f\left(x-x^{\prime}\right)=\cos \left(x-x^{\prime}\right) . H_{1}, H_{2}$ are independent matrices belonging to the appropriate GE. Fig. 1 shows the results for both the GOP and GUP. We find that $c_{\Gamma}\left(x-x^{\prime}\right)$ is approximated very well by

$$
c_{\Gamma}\left(\bar{x}-\bar{x}^{\prime}\right)=\left[\frac{1}{1+\left(\bar{x}-\bar{x}^{\prime}\right)^{2} / \alpha_{\beta}^{2}}\right]^{\beta},
$$


namely a Lorentzian in the orthogonal case and a squared Lorentzian in the unitary case. Using 300 simulations with $N=150$ and estimating the statistical error at each $x$, a leastsquares fit to (32) gives $\alpha_{1}=0.48 \pm 0.04$ and $\alpha_{2}=0.64 \pm 0.04$. The numerical simulations of the correlators (diamonds) and their best fit to (32) (dashed) are shown in Fig. 11. We have also confirmed in our simulations the independence of the details of the channels by computing the correlator (22) with various sets of channel vectors $\phi_{c}$ and verifying the agreement with (32). Comparing the orthogonal and unitary results shows that breaking time-reversal symmetry accelerates the decorrelation. In Section IV.C we use perturbation theory to derive an exact analytic expression for $c_{\Gamma}$ to leading order in $\Delta \bar{x}$. This leading order differs from that of (32), and thus expression (32) is just an approximation.

To test the universality of the conductance correlator, we model a disordered dot by the two-dimensional Anderson Hamiltonian [35,36]

$$
H=\sum_{\alpha}\left(u_{\alpha}+w_{\alpha}\right) a_{\alpha}^{\dagger} a_{\alpha}-\sum_{\langle\alpha \beta\rangle} e^{i \theta_{\alpha \beta}} a_{\alpha}^{\dagger} a_{\beta}
$$

where the second sum is over nearest-neighbors lattice sites only. The on-site energies $w_{\alpha}$ are distributed uniformly over the interval $[-W / 2, W / 2]$. The $u_{\alpha}$ describes the non-random component of the potential whereas the phases $\theta_{\alpha \beta}$ are determined by the magnetic flux. We use a $n_{x} \times n_{y}=27 \times 27$ lattice folded into a cylinder whose symmetry axis lies along $x$, imposing appropriate boundary conditions. The left and right leads are represented by arrays $A^{l, r}$ of $m_{1} \times m_{2}$ lattice points with total widths given by $\Gamma_{\lambda}^{i}=\sum_{\vec{r}_{\alpha} \in A^{i}}\left(v_{\alpha}^{i}\right)^{2}\left|\Psi_{\lambda}\left(\vec{r}_{\alpha}\right)\right|^{2}$ such that each point contact $\vec{r}_{\alpha}$ constitutes a channel with coupling $\left(v_{\alpha}^{i}\right)^{2}$ [30].

We introduce a parametric dependence by applying a step potential along $x, u(x, y)=$ $u_{0} \Theta(x)$, using its strength $u_{0}$ as the parameter. The results for the conductance correlator, which are presented in Fig. 1 (left) for $2 \times 2$ leads and $v_{\alpha}^{2}=1$, are in excellent agreement with the GOP prediction (32) (with $\beta=1$ ).

We break time-reversal symmetry by applying a magnetic field $B$ pointing along the cylinder axis, corresponding to $\theta_{\alpha \beta}=\frac{2 \pi}{n_{y}} \Phi / \Phi_{0}$ in (33) if $\vec{r}_{\alpha}, \vec{r}_{\beta}$ are neighbors along $y$ and $\theta_{\alpha \beta}=0$ otherwise, where $\Phi / \Phi_{0}$ is the magnetic flux associated with $B$ in units of the flux quantum $\Phi_{0}=h c / e$. We take $\Phi / \Phi_{0}=1 / 4$ to achieve maximal symmetry breaking. The parametric dependence is introduced through a step potential and the results are presented in Fig. 1 (right) using the same lead arrangements as above. The agreement with the GUP prediction (32) (with $\beta=2$ ) is again very good.

We remark that the partial width is analogous to the eigenfunction intensity at a fixed spatial point $\vec{r}$. It follows that the parametric eigenfunction intensity correlator in a chaotic system is identical to the width correlator $c_{\Gamma}(30)$. Denoting by $\Psi^{x}(\vec{r})$ the eigenfunction at $\vec{r}$ for a value $x$ of an external parameter, we have

$$
\overline{\tilde{I}(x) \tilde{I}\left(x^{\prime}\right)}=c_{\Gamma}\left(x-x^{\prime}\right), \quad I(x)=\left|\Psi^{x}(\vec{r})\right|^{2} .
$$

The eigenfunction intensities are measurable in microwave cavity experiments [22,23] and our predictions for $c_{\Gamma}\left(x-x^{\prime}\right)$ can be tested there as well. 


\section{Relation to the Parametric Overlap Correlator}

It is interesting to note that the result (32) is identical (within statistical errors) to our result for the universal form of the averaged parametric overlap [15]

$$
o\left(x-x^{\prime}\right)=\overline{\left|\left\langle\psi_{\lambda}(x) \mid \psi_{\lambda}\left(x^{\prime}\right)\right\rangle\right|^{2}} .
$$

We now derive an exact relation between the width and overlap correlators (for finite $N$ ) and show that they are identical in the limit $N \rightarrow \infty$. Choosing a complete set of vectors $\hat{\phi}_{i} ; i=1, \ldots, N$ the overlap correlator can be written as

$$
o\left(x-x^{\prime}\right)=\sum_{i} \overline{\left|\hat{\gamma}_{i}(x)\right|^{2}\left|\hat{\gamma}_{i}\left(x^{\prime}\right)\right|^{2}}+\sum_{i \neq j} \overline{\hat{\gamma}_{i}^{*}(x) \hat{\gamma}_{j}(x) \hat{\gamma}_{i}\left(x^{\prime}\right) \hat{\gamma}_{j}^{*}\left(x^{\prime}\right)}
$$

Since the various correlators in (36) are independent of the particular channel $i$ or the pair $i, j$ of orthogonal channels we can rewrite it as

$$
o\left(x-x^{\prime}\right)=N\left(\overline{\hat{\Gamma}_{1}(x) \hat{\Gamma}_{1}\left(x^{\prime}\right)}-{\overline{\hat{\Gamma}_{1}}}^{2}\right)+\frac{N(N-1)}{2}\left[\overline{\hat{\gamma}_{1}^{*}(x) \hat{\gamma}_{2}(x) \hat{\gamma}_{1}\left(x^{\prime}\right) \hat{\gamma}_{2}^{*}\left(x^{\prime}\right)}+\text { c.c. }\right]+\frac{1}{N} .
$$

To proceed we need to use a relation between the two parametric correlators on the r.h.s. of (37), the width correlator and the "exchange" correlator:

$$
\frac{1}{2}\left[\overline{\hat{\gamma}_{1}^{*}(x) \hat{\gamma}_{2}(x) \hat{\gamma}_{1}\left(x^{\prime}\right) \hat{\gamma}_{2}^{*}\left(x^{\prime}\right)}+\text { c.c. }\right]=\frac{\beta}{2} \frac{N}{N-1}\left[\overline{\hat{\Gamma}_{1}(x) \hat{\Gamma}_{1}\left(x^{\prime}\right)}-{\overline{\hat{\Gamma}_{1}}}^{2}\right]
$$

At $x=x^{\prime}$, this relation is easily derived using the results of Appendix A, and for small $x-x^{\prime} \neq 0$, it can be proven perturbatively (see Appendix B). At the end of this section we provide a proof of this non-trivial relation for any $x, x^{\prime}$. Using (38) in (37) we find

$$
o\left(x-x^{\prime}\right)=N \frac{\beta N+2}{2}\left[\overline{\hat{\Gamma}_{1}(x) \hat{\Gamma}_{1}\left(x^{\prime}\right)}-{\overline{\hat{\Gamma}_{1}}}^{2}\right]+\frac{1}{N} .
$$

Finally, using the GE relation $\bar{\Gamma}_{1}^{2}-{\overline{\hat{\Gamma}_{1}}}^{2}=2(N-1) / N^{2}(\beta N+2)$ (see Appendix A), we find the exact relation between the overlap and width correlator

$$
o\left(x-x^{\prime}\right)=\left(1-\frac{1}{N}\right) c_{\Gamma}\left(x-x^{\prime}\right)+\frac{1}{N} .
$$

In the limit $N \rightarrow \infty$ these two correlators become identical.

We now return to prove (38). We first rederive the independence of the width correlator of the matrix $M$ in a different way. The channel vectors $\phi_{c}$ can be transformed by a linear transformation $\phi_{c}=\sum_{c^{\prime}} \hat{\phi}_{c^{\prime}} F_{c^{\prime} c}$ to orthonormal channels $\left\langle\hat{\phi}_{c} \mid \hat{\phi}_{c^{\prime}}\right\rangle=\delta_{c c^{\prime}}$. In terms of $F$, the original channel matrix is $\left\langle\phi_{c} \mid \phi_{c^{\prime}}\right\rangle=\left(F^{\dagger} F\right)_{c c^{\prime}}$. A simple solution for $F$ is $F=(N W)^{1 / 2} U$ where $U$ is the unitary matrix that diagonalizes $M$ and $W$ is the diagonal matrix composed of the eigenvalues $w_{c}^{2}$ of $M$ (see previous section). In this case $F F^{\dagger}=N W$ is diagonal. However, we can take advantage of the freedom to make a unitary transformation $R$ on the orthonormal eigenchannels of the previous section to obtain another orthonormal set $\hat{\phi}_{c}$. This leads to $F=R(N W)^{1 / 2} U$ which in general has a non-diagonal $F F^{\dagger}=N R W R^{\dagger}$. 
Calculating the width correlator directly from the expression $\Gamma=\hat{\gamma}^{\dagger} F F^{\dagger} \hat{\gamma}$ for the total width, we now find

$$
\begin{aligned}
\overline{\Gamma(x) \Gamma\left(x^{\prime}\right)}-\bar{\Gamma}^{2}= & \left\{\sum_{c}\left[\left(F F^{\dagger}\right)_{c c}\right]^{2}\right\}\left(\overline{\hat{\Gamma}_{1}(x) \hat{\Gamma}_{1}\left(x^{\prime}\right)}-{\overline{\hat{\Gamma}_{1}}}^{2}\right) \\
& +\frac{1}{\beta}\left\{\sum_{c \neq c^{\prime}}\left|\left(F F^{\dagger}\right)_{c c^{\prime}}\right|^{2}\right\}\left[\overline{\hat{\gamma}_{1}^{*}(x) \hat{\gamma}_{2}(x) \hat{\gamma}_{1}\left(x^{\prime}\right) \hat{\gamma}_{2}^{*}\left(x^{\prime}\right)}+c . c .\right] \\
& +\left\{\sum_{c \neq c^{\prime}}\left(F F^{\dagger}\right)_{c c}\left(F F^{\dagger}\right)_{c^{\prime} c^{\prime}}\right\}\left(\overline{\hat{\Gamma}_{1}(x) \hat{\Gamma}_{2}\left(x^{\prime}\right)}-\overline{\hat{\Gamma}_{1} \hat{\Gamma}_{2}}\right),
\end{aligned}
$$

The third term on the r.h.s. of (41)) is just the cross-channel correlator and according to (27) can be combined with the autochannel width correlator (the first term on the r.h.s. of (41)). Notice that for the choice made of $F$ in the previous section $\left(F F^{\dagger}=W\right.$ diagonal $)$ the second term on the r.h.s. of (41) vanishes, unlike the present case. However, since one must recover the same width correlator in (26) and in (41), we conclude that the first two correlators on the r.h.s. of (41) must be related. Indeed, if we assume relation (38) to hold, the two terms in (41) combine to give

$$
\overline{\Gamma(x) \Gamma\left(x^{\prime}\right)}-\bar{\Gamma}^{2}=\left[\operatorname{Tr}\left(F F^{\dagger}\right)^{2}-\frac{1}{N-1}\left(\left(\operatorname{Tr} F F^{\dagger}\right)^{2}-\operatorname{Tr}\left(F F^{\dagger}\right)^{2}\right)\right]\left[\overline{\hat{\Gamma}_{1}(x) \hat{\Gamma}_{1}\left(x^{\prime}\right)}-{\overline{\hat{\Gamma}_{1}}}^{2}\right] .
$$

Since $\operatorname{Tr}\left(F F^{\dagger}\right)^{2}=\operatorname{Tr} M^{2}=\sum_{c} w_{c}^{4}$ and $\operatorname{Tr}\left(F F^{\dagger}\right)=\operatorname{Tr} M$, Eq. (42) is then identical to (29).

\section{THE ROTATION SCALING}

Although all parametric correlators become universal upon the diffusion scaling (20), performing this scaling is not always feasible. In particular, the scaling factor $D$ is not directly accessible in quantum dot experiments. As a result of the Coulomb blockade suppression of tunneling, the energy levels probed as the gate voltage is varied are the ground states for successive electron numbers rather than the excited states for a fixed electron number. Therefore one can measure the charging energy $e^{2} / C$ but not the level spacing $\Delta$ which is necessary to determine $D$. While in principle it is possible to measure the variation of the ground state energy of the dot as a function of $x$ by tracing the peak amplitude, the mean level spacing $\Delta$ can only be estimated theoretically from e.g. a Fermi gas model. Since the interaction and the dot's shape affect $\Delta$, it is difficult to extract accurate values for $D$ from the data.

In this section we introduce a new scaling which is calculated from the eigenfunctions alone, or alternatively from the level widths. This makes it possible to extract the scaled parameter directly from the conductance data (see also Sec. VI.C), so that our predictions for $c_{G}\left(\bar{x}-\bar{x}^{\prime}\right)$ could be tested experimentally.

\section{A. Eigenfunction Rotation Rate and Parameter Scaling}

We consider the quantity $\overline{\left|\Delta \gamma_{\lambda}\right|^{2}} \equiv \overline{\left|\gamma_{\lambda}\left(x^{\prime}\right)-\gamma_{\lambda}(x)\right|^{2}}$ where $\gamma_{\lambda}(x)=\left\langle\phi \mid \psi_{\lambda}(x)\right\rangle$ is the partial level width and $\phi$ is a channel vector which we take for convenience to be normalized. 
This quantity is independent of our choice for $\phi$ since the GOP (GUP) is invariant under an $x$-independent orthogonal (unitary) transformation that will transform $\phi$ into another normalized vector. From first-order perturbation theory we have

$$
\Delta \gamma_{\lambda}=\gamma_{\lambda}\left(x^{\prime}\right)-\gamma_{\lambda}(x)=\sum_{\mu \neq \lambda} \frac{\left\langle\psi_{\mu}(x)\left|H\left(x^{\prime}\right)\right| \psi_{\lambda}(x)\right\rangle}{E_{\lambda}(x)-E_{\mu}(x)} \gamma_{\mu}(x)+i \omega_{\lambda}(x) \gamma_{\lambda}(x) \Delta x
$$

where $\omega_{\lambda}(x)$ is an arbitrary real function that corresponds to the freedom we have in choosing the phase of $\psi_{\lambda}$. We first make the usual choice $\omega_{\lambda}=0$, but we shall see below that another choice would be useful for computational purposes. The variance of $\Delta \gamma_{\lambda}$ is calculated in two steps. First, we average over $H\left(x^{\prime}\right)$ keeping $H(x)$ fixed using the conditional two-matrix distribution [15]

$$
\begin{array}{r}
P\left[H\left(x^{\prime}\right) \mid H(x)\right]=P\left[H(x), H\left(x^{\prime}\right)\right] / P[H(x)] \\
\propto \exp \left\{-\frac{\beta}{2 a^{2}} \frac{1}{1-f^{2}} \operatorname{Tr}\left[H\left(x^{\prime}\right)-f H(x)\right]^{2}\right\}
\end{array}
$$

with $f \equiv f\left(x-x^{\prime}\right)$. We can write the conditional averages as

$$
\begin{aligned}
\overline{H_{\mu \lambda}^{\prime}-f H_{\mu \lambda}} & =0 \\
\overline{\left(H_{\mu \lambda}^{\prime}-f H_{\mu \lambda}\right)\left(H_{\sigma \eta}^{\prime}-f H_{\sigma \eta}\right)} & =\frac{a^{2}}{2 \beta} g_{\mu \lambda, \sigma \eta}^{(\beta)}\left(1-f^{2}\right) .
\end{aligned}
$$

Here and in the following we use the notation $H_{\mu \lambda}=\left\langle\psi_{\mu}|H| \psi_{\lambda}\right\rangle$ and $H_{\mu \lambda}^{\prime}=\left\langle\psi_{\mu}\left|H^{\prime}\right|\right.$ $\left.\psi_{\lambda}\right\rangle$, where matrix elements are calculated in the basis of the eigenstates of $H(x)$. Primed quantities take their value at $x^{\prime}$ whereas non-primed quantities take their value at $x$. To do the conditional averaging we write $\left|\Delta \gamma_{\lambda}\right|^{2}$ in the form

$$
\left|\Delta \gamma_{\lambda}\right|^{2}=\sum_{\mu \nu \neq \lambda} \frac{\left(H_{\mu \lambda}^{\prime}-f H_{\mu \lambda}\right)\left(H_{\nu \lambda}^{\prime *}-f H_{\nu \lambda}^{*}\right)}{\left(E_{\lambda}-E_{\mu}\right)\left(E_{\lambda}-E_{\nu}\right)} \gamma_{\mu} \gamma_{\nu}^{*}
$$

which is convenient since $P\left[H^{\prime} \mid H\right]$ depends on $H^{\prime}-f H$ (note that $H_{\mu \lambda}=0$ ). After averaging over $H^{\prime}$ we get

$$
\overline{\left|\Delta \gamma_{\lambda}\right|^{2}}=\frac{a^{2}}{2 \beta}\left(1-f^{2}\right) \overline{\sum_{\mu \neq \lambda} \frac{\left|\gamma_{\mu}\right|^{2}}{\left(E_{\lambda}-E_{\mu}\right)^{2}}} .
$$

Next we perform the average over $H$. Since the joint eigenvalue and eigenfunction distribution factorizes in the GE [5] and $\overline{\left|\gamma_{\mu}\right|^{2}}$ is $\mu$-independent we have, using the short-distance expansion of $f\left(x-x^{\prime}\right)$ (17),

$$
\overline{\left|\Delta \gamma_{\lambda}\right|^{2}}=\beta \overline{\left|\gamma_{\lambda}\right|^{2}} N Z \kappa|\Delta x|^{\eta}
$$

where

$$
Z \equiv \frac{a^{2}}{\beta N} \overline{\sum_{\mu \neq \lambda} \frac{1}{\left(E_{\lambda}-E_{\mu}\right)^{2}}}
$$


Dyson [37 obtained an exact result for a related quantity,

$$
Z_{D}=\frac{a^{2}}{\beta N} \frac{1}{N_{\lambda, \mu \neq \lambda} \frac{1}{\left(E_{\lambda}-E_{\mu}\right)^{2}}}=\frac{1-\frac{1}{N}}{2(\beta-1)} .
$$

However, $E_{\lambda}$ in (49) is near the middle of the spectrum where the level density is roughly constant whereas in (50) it ranges over the whole semi-circle, hence $Z \neq Z_{D}$. It is more suitable for our purpose to unfold the levels and approximate the sum in (49) using the two-point cluster function $Y_{2}(\epsilon)$ [5],

$$
Z=\frac{a^{2}}{\beta N} \frac{1}{\Delta^{2}} \overline{\sum_{\mu \neq \lambda} \frac{1}{\left(\varepsilon_{\lambda}-\epsilon_{\mu}\right)^{2}}} \approx \frac{4}{\pi^{2} \beta} \int_{0}^{\infty} \frac{d \varepsilon}{\epsilon^{2}}\left[1-Y_{2}(\varepsilon)\right] .
$$

The integral diverges for $\beta=1$, as does Dyson's result (50). For $\beta=2$ it can be calculated using the simple Fourier representation $Y_{2}(\varepsilon)=\sin ^{2}(\pi \varepsilon) /(\pi \varepsilon)^{2}=\int_{-1}^{1} d k e^{-2 \pi i k \varepsilon}(1-|k|)$, giving $Z=2 / 3$ compared to Dyson's $Z_{D}=1 / 2$.

Eq. (48) can be rewritten (for $\Delta x \rightarrow 0$ ) as an amplitude diffusion law

$$
\overline{\left|\Delta \gamma_{\lambda}\right|^{2}} / \overline{\left|\gamma_{\lambda}\right|^{2}}=\beta R|\Delta x|^{\eta}+\ldots
$$

in analogy with the level diffusion law (18), where

$$
R \equiv \lim _{\Delta x \rightarrow 0} \frac{1}{\beta \overline{\left|\gamma_{\lambda}\right|^{2}}} \frac{\overline{\left|\Delta \gamma_{\lambda}\right|^{2}}}{|\Delta x|^{\eta}}
$$

In the GP we have $R=\kappa N Z$. Eq. (52) motivates the parameter scaling

$$
x \rightarrow \bar{x}_{r}=R^{1 / \eta} x
$$

which we call the rotation scaling as explained below. Under this scaling, just like the diffusion scaling (20), the process correlation function (17) becomes

$$
f \approx 1-\frac{1}{N Z}\left|\bar{x}_{r}-\bar{x}_{r}^{\prime}\right|^{\eta}
$$

and since all correlators depend on $N(1-f)$ rather than on $N$ and $f$ separately [15], we conclude that all correlators are universal functions of $\left|\bar{x}_{r}-\bar{x}_{r}^{\prime}\right|^{\eta}$. We remark that $R$ can also be written as $R=\beta^{-1} \lim _{\Delta x \rightarrow 0} \overline{\left|\Delta \tilde{\gamma}_{\lambda}\right|^{2}} /|\Delta x|^{\eta}$ where $\tilde{\gamma}_{\lambda} \equiv \gamma_{\lambda} / \sqrt{\overline{\left|\gamma_{\lambda}\right|^{2}}}$ is an "unfolded" amplitude in analogy with the unfolded level used in the definition (19).

For the case of broken time-reversal symmetry (GUP), the amplitude $\gamma_{\lambda}$ is generally complex when evaluated according to (43) with $\omega_{\lambda}=0$. Note, however, that the eigenfunctions $\psi_{\lambda}(x)$ are determined up to an (x-dependent) phase, and we can choose this phase such that the amplitude $\gamma_{\lambda}=\left\langle\phi \mid \psi_{\lambda}\right\rangle$ is real for any $x$. For this choice to be consistent we must fix $\omega_{\lambda}(x)$ such that $\omega_{\lambda}(x) \gamma_{\lambda}(x) \Delta x$ cancels the imaginary part of the sum on the r.h.s. of (43), giving 


$$
\Delta \gamma_{\lambda}=\sum_{\mu \neq \lambda} \frac{\operatorname{Re} H_{\mu \lambda}^{\prime}}{E_{\lambda}-E_{\mu}} \gamma_{\mu}
$$

which is real as well. Since only the real part of the matrix elements of $H$ is used in (56), the expression (53) for $R$ is now replaced by

$$
R \equiv \lim _{\Delta x \rightarrow 0} \frac{1}{\overline{\gamma_{\lambda}^{2}}} \frac{\overline{\left(\Delta \gamma_{\lambda}\right)^{2}}}{|\Delta x|^{\eta}}
$$

In practice it is more convenient to use this definition, according to which $\gamma_{\lambda}=\sqrt{\Gamma_{\lambda}}$ and is thus real.

In most physical applications, the dependence of $H$ on $x$ is analytic and the GP must be differentiable, i.e $\eta=2$ [16]. The scaling factor is then given by

$$
R=\frac{1}{\beta \overline{\left|\gamma_{\lambda}\right|^{2}}} \overline{\left|\frac{\partial \gamma_{\lambda}}{\partial x}\right|^{2}},
$$

which can be interpreted as the eigenfunction rotation rate. To see this we note that as $x$ increases, the eigenfunctions rotate in the Hilbert space and this rotation is characterized by an anti-Hermitian operator $\Omega(x)$

$$
\frac{\partial}{\partial x}\left|\psi_{\lambda}\right\rangle=\Omega\left|\psi_{\lambda}\right\rangle
$$

whose matrix elements in the basis $\psi_{\lambda}$ are given by $\Omega_{\mu \lambda}=\left\langle\psi_{\mu} \mid \partial \psi_{\lambda} / \partial x\right\rangle=\left(1-\delta_{\mu \lambda}\right) \frac{\left(\partial H_{\mu \lambda} / \partial x\right)}{E_{\lambda}-E_{\mu}}$ (see (43)). The basis-independent quantity $N^{-1} \overline{\operatorname{Tr}\left(\Omega^{\dagger} \Omega\right)}$ is obtained from (59) with the help of a complete set of orthonormal vectors $\phi_{\mu}$

$$
\frac{1}{N} \overline{\operatorname{Tr}\left(\Omega^{\dagger} \Omega\right)}=\frac{1}{N} \sum_{\lambda \mu} \overline{\left|\left\langle\phi_{\mu} \mid \partial \psi_{\lambda} / \partial x\right\rangle\right|^{2}}=R
$$

exploiting the $\phi_{\mu}$-independence of the average in the sum in (60) and the fact that $\overline{\gamma_{\lambda}^{2}}=$ $1 / N$. $R$ is therefore the variance of the matrix elements of the scaled rotation rate matrix $\sqrt{N} \Omega(x)=\Omega(x) / \sqrt{\overline{\left|\gamma_{\lambda}\right|^{2}}}$, in analogy with $D$ being the variance of the scaled level velocity $\left(\partial E_{\lambda} / \partial x\right) / \Delta$. It also follows from (59) that $R$ is the norm of the eigenfunction derivative,

$$
R=\overline{\left\langle\partial \psi_{\lambda} / \partial x \mid \partial \psi_{\lambda} / \partial x\right\rangle}
$$

If the system obeys the GE statistics at each $x$, then $\overline{\partial \gamma_{\lambda} / \partial x}=0$ and the quantity $\overline{\left|\partial \gamma_{\lambda} / \partial x\right|^{2}}$ in the definition of $R$ gives the variance of $\partial \gamma_{\lambda} / \partial x$. This should be contrasted with the level velocity scaling $D$, where the physical system can have a drift such that $\overline{\partial \epsilon_{\lambda} / \partial x} \neq 0$ which has to be removed.

While the values of the scaling factors $D$ and $R$ are system-dependent, the ratio $R / D$ is universal and is given by

$$
R / D=\frac{\beta}{4} \pi^{2} Z
$$


For the GUP $Z=2 / 3$ and

$$
R / D=\frac{\pi^{2}}{3} \quad(G U P) .
$$

If we use Dyson's $Z_{D}$ in (63), we obtain $R / D=\pi / 2$. The results from simulations are in clear agreement with $R / D=\pi^{2} / 3$, as can be seen in Fig. 2.

It is important to note that $R$ diverges in the orthogonal case (in the limit $\Delta x \rightarrow 0$ ) because of the divergence of $Z$ in $(50,51)$ and the rotation scaling, as defined above, is valid for the unitary case only. However, it is possible to fix this flaw as we discuss in the next section.

\section{B. Rotation Scaling in the GOP}

We now analyze the divergence of $R$ in the orthogonal case and show that it is nevertheless possible to use it to perform the scaling. We note that $\overline{\left|\Delta \gamma_{\lambda}\right|^{2}} / \overline{\left|\gamma_{\lambda}\right|^{2}}$ is bounded from above by 4 and is therefore finite for any $\Delta x$. This is in contradiction to (52), according to which this quantity diverges for any fixed $\Delta x>0$. This indicates that while the perturbation expansion (43) is valid for a given member of the ensemble (for $\Delta x$ sufficiently small), the ensemble average of the leading order term diverges due to contributions from members with small level spacings. Nevertheless, since $\overline{\left|\Delta \gamma_{\lambda}\right|^{2}} / \overline{\left|\gamma_{\lambda}\right|^{2}}$ is finite, we can define a scaling factor $\tilde{R}(\Delta x)$ (in analogy with (53)) for any finite value of $\Delta x \neq 0$ by

$$
\tilde{R}(\Delta x) \equiv \frac{1}{\beta \overline{\left|\gamma_{\lambda}\right|^{2}}} \frac{\overline{\left|\Delta \gamma_{\lambda}\right|^{2}}}{|\Delta x|^{\eta}} .
$$

$\tilde{R}$ diverges (in the GOP) as $\Delta x \rightarrow 0$. From $R=\kappa N Z$, it is clear that this divergence is caused by the small level spacings $s=\left(E_{\lambda}-E_{\mu}\right) / \Delta$ which are distributed according to $p(s) \propto s^{\beta}$ for small $\mathrm{s}$ [司]. Indeed $\int d s s^{\beta-2}$ converges near $s=0$ for $\beta>1$ but diverges logarithmically for $\beta=1$. The expression (51) for $Z$ can be regularized by introducing a small spacing cut-off at $\varepsilon=\delta$. From $Y_{2}(\varepsilon) \approx 1-\pi^{2} \varepsilon / 6+\mathcal{O}\left(\varepsilon^{3}\right)$ [5], we find

$$
Z_{\delta}=-\frac{2}{3} \ln \delta+\mathcal{O}(1) .
$$

To regularize the perturbative expression (48) we examine the conditional average for 1 $\left.\Delta \gamma_{\lambda}\right|^{2}$ at fixed $H(x)$ (see (47))

$$
\left.\overline{\left|\Delta \gamma_{\lambda}\right|^{2}}\right|_{\text {fixed } H(x)}=\frac{1}{2} \sum_{\mu \neq \lambda} \frac{\left|\gamma_{\mu}\right|^{2}}{\left(\varepsilon_{\lambda}-\varepsilon_{\mu}\right)^{2}}|\Delta \bar{x}|^{\eta},
$$

written in terms of the unfolded energies and the scaled parameter. This expression is welldefined for any given member $H(x)$ of the GE ensemble at $x$. However, for a member with a level spacing that is too small, the approximation (66), which is of the order $|\Delta x|^{\eta}$, breaks down unless we also make $|\Delta x|^{\eta}$ smaller. Consequently, the average over all $H(x)$ at fixed $|\Delta x|^{\eta}$ leads to divergence. To avoid that, we use a small spacing cut-off $\delta$ (as for $Z$ above) 
that depends on $\Delta x$. Noting that the parameter which determines the goodness of the approximation (66) is $|\Delta x|^{\eta} / \delta^{2}$, we make the conjecture that a regularized perturbative expansion can be obtained by assuming $\delta^{2} \propto|\Delta \bar{x}|^{\eta}$. This is equivalent to relating two (unfolded) energy scale $\delta^{2} \propto \overline{(\Delta \varepsilon)^{2}}$ where the latter is associated with a change $\Delta \bar{x}$ in the scaled external parameter. This regularization leads to

$$
\tilde{R}=\kappa N\left(-\frac{1}{3} \ln |\Delta \bar{x}|^{\eta}+\mathcal{O}(1)\right)
$$

Our conjecture is supported by an exact result for $N=2$ which exhibits this behavior (see Section VII), and is confirmed numerically for large $N$ below. In analogy with the universal ratio $R / D$ for the GUP (63) we now have a universal ratio for the GOP (for small $\Delta \bar{x}$ )

$$
\frac{\tilde{R}}{D}=-\frac{\pi^{2} \eta}{12} \ln (\Delta \bar{x})+\text { Const. } \quad(G O P)
$$

Eq. (68) can be used to determine the scaling factor $D$ (in cases it is immeasurable) from the measured $\tilde{R}$.

Fig. 2 (left panel) presents the scaling ratio $\tilde{R} / D$ computed with a finite $\Delta x$ as a function of $\ln \Delta \bar{x}$. Shown are results of GP and Anderson model simulations compared to our conjecture for the orthogonal case (68). Similar calculations are shown (right panel) for the unitary case as a function of $\Delta x$ and they converge to (63) in the limit $\Delta x \rightarrow 0$. The agreement is very good. We remark that the smaller $\Delta x$ is, the more simulations should be performed to get good statistics due to increasing fluctuations. Consequently, the GOP divergence would be difficult to observe experimentally.

\section{Perturbative Calculation of the Width Correlator}

We now calculate $c_{\Gamma}\left(x-x^{\prime}\right)$ for a symmetric dot perturbatively to leading order. A convenient way to calculate the width correlator is to use the relation

$$
c_{\Gamma}\left(x-x^{\prime}\right)=1-\frac{1}{2} \frac{\overline{\left(\Delta \Gamma_{\lambda}\right)^{2}}}{\overline{\Gamma_{\lambda}^{2}}-\bar{\Gamma}_{\lambda}^{2}},
$$

where $\Delta \Gamma_{\lambda} \equiv \Gamma_{\lambda}\left(x^{\prime}\right)-\Gamma_{\lambda}(x)$. Using $\Gamma_{\lambda}\left(x^{\prime}\right)=\left|\gamma_{\lambda}\left(x^{\prime}\right)\right|^{2}$ in (43) we get

$$
\Gamma_{\lambda}^{\prime}=\Gamma_{\lambda}+\sum_{\mu \neq \lambda}\left(\frac{H_{\mu \lambda}^{\prime}}{E_{\lambda}-E_{\mu}} \gamma_{\lambda}^{*} \gamma_{\mu}+\text { c.c. }\right)+\ldots .
$$

After averaging

$$
\left(\Gamma_{\lambda}^{\prime}-\Gamma_{\lambda}\right)^{2}=\sum_{\mu \nu \neq \lambda} \frac{H_{\mu \lambda}^{\prime} H_{\nu \lambda}^{\prime *}}{\left(E_{\lambda}-E_{\mu}\right)\left(E_{\lambda}-E_{\nu}\right)} \gamma_{\mu} \gamma_{\nu}^{*} \Gamma_{\lambda}+\text { c.c. }
$$

over $H\left(x^{\prime}\right)$ while keeping $H(x)$ fixed, similarly to the calculation (46), we are left with

$$
\overline{\left(\Gamma_{\lambda}^{\prime}-\Gamma_{\lambda}\right)^{2}}=\frac{2 a^{2}}{\beta}\left(1-f^{2}\right) \overline{\sum_{\mu \neq \lambda} \frac{1}{\left(E_{\lambda}-E_{\mu}\right)^{2}} \Gamma_{\mu} \Gamma_{\lambda}}
$$


where the average is over $H(x)$. Eqs. (17,49, 53) then give

$$
\overline{\left(\Gamma_{\lambda}^{\prime}-\Gamma_{\lambda}\right)^{2}}=4 \overline{\Gamma_{\mu} \Gamma_{\lambda}} R|\Delta x|^{\eta} \text {. }
$$

Using the GE relation $\overline{\Gamma_{\mu} \Gamma_{\lambda}}=\frac{\beta}{2} \frac{N}{N-1}\left(\overline{\Gamma_{\lambda}^{2}}-{\overline{\Gamma_{\lambda}}}^{2}\right.$ ) (valid for $\mu \neq \lambda$ ) together with (69) we obtain in the level velocity scaling

$$
c_{\Gamma}\left(x-x^{\prime}\right)=1-\beta \frac{N}{N-1} \frac{R}{D}\left|\bar{x}-\bar{x}^{\prime}\right|^{\eta} .
$$

For the GUP we use Eq. (63) to find

$$
c_{\Gamma}\left(x-x^{\prime}\right) \approx 1-\frac{2 \pi^{2}}{3}\left|\bar{x}-\bar{x}^{\prime}\right|^{\eta}
$$

as $N \rightarrow \infty$. When we compare (75) with the leading-order behavior of the squared Lorentzian (32) for the GUP case we find $\alpha_{2}=\sqrt{3} / \pi \approx 0.55$. The discrepancy with the least-square fit value quoted below (32) indicates that (32), while being a good approximation, is not exact. Indeed, including higher-order terms in the perturbative calculation introduces odd powers of $\left|\bar{x}-\bar{x}^{\prime}\right|^{\eta / 2}$ into $c_{\Gamma}$ (see Section V.D).

In the GOP the perturbative approximation (53) diverges. Using a regularization similar to that invoked in the previous section amounts to replacing $R$ in (53) by $\tilde{R}$ as given by (67). Using (68) this leads to the following short-distance behavior of the GOP conductance correlator

$$
c_{\Gamma}\left(x-x^{\prime}\right) \approx 1-\frac{N}{N-1}\left|\bar{x}-\bar{x}^{\prime}\right|^{\eta}\left(-\frac{\pi^{2} \eta}{12} \ln \left|\bar{x}-\bar{x}^{\prime}\right|+\text { Const. }\right) .
$$

This non-analyticity near the origin indicates that the Lorentzian (32) which provides a very good approximation for $c_{\Gamma}\left(x-x^{\prime}\right)$ is not the exact result. We remark that the $N=2$ case exhibits the same behavior (see Section VII).

\section{The Rotation Scaling as the RMS Width Velocity}

In Ref. [15] we have shown that the quantity $\overline{\left(\Delta \varepsilon_{\lambda}\right)^{2}}$, which measures the diffusion of the unfolded energy levels in $x$, is a universal function of $(\Delta \bar{x})^{\eta}$ (even when the latter is not small). Similarly, one can consider the quantity $\overline{\left(\Delta \Gamma_{\lambda}\right)^{2}} / \bar{\Gamma}_{\lambda}^{2}$ where $\Delta \Gamma_{\lambda} \equiv \Gamma_{\lambda}\left(x^{\prime}\right)-\Gamma_{\lambda}(x)$ as a measure of the diffusion of the "unfolded" widths $\Gamma_{\lambda} / \bar{\Gamma}_{\lambda}$. Based on our discussion from Section III.A, this must also be a universal function of $(\Delta \bar{x})^{\eta}$. We have computed this function for the GOP and GUP using the simple GP $\cos x H_{1}+\sin x H_{2}$, and it is shown in Fig. 3.

Using the perturbative expression (73), we find that for small $\Delta x$

$$
\frac{1}{\bar{\Gamma}_{\lambda}^{2}} \overline{\left(\Delta \Gamma_{\lambda}\right)^{2}}=4 \frac{N}{N+\beta / 2} \tilde{R}|\Delta x|^{\eta} \approx 4 \frac{\tilde{R}}{D}|\Delta \bar{x}|^{\eta} .
$$

For the GUP we expect to find $\tilde{R} / D \rightarrow R / D=\pi^{2} / 3$ in the limit $\Delta x \rightarrow 0$, while for the GOP $\tilde{R} / D$ should have the logarithmic universal behavior (68) at small $\Delta x$. 
Eq. (77) leads us to an alternative definition of the rotation scaling

$$
\tilde{R} \equiv \frac{1}{4 \bar{\Gamma}_{\lambda}^{2}} \frac{\overline{\left|\Delta \Gamma_{\lambda}\right|^{2}}}{|\Delta x|^{\eta}} .
$$

In most physical applications, the parametric dependence is analytic so $\eta=2$, and the scaling factor $\sqrt{R}$ is just the RMS of the rate of change of the normalized width as a function of the parameter

$$
R=\frac{1}{4 \bar{\Gamma}_{\lambda}^{2}} \overline{\left(\frac{\partial \Gamma_{\lambda}}{\partial x}\right)^{2}}
$$

We remark that the definition (79) of the rotation scaling has the advantage that there is no phase ambiguity in $\Gamma(x)$. Furthermore, in quantum dots experiments, the measurable quantity is the width (i.e. conductance for a symmetric dot), so (79) allows us to extract the scaled parameter directly from the data.

It is possible to calculate perturbatively higher order terms in (77). In the GUP, while the leading order converges, the next order does not. For example, one of the terms of order $\Delta x^{2 \eta}$ includes a diverging GUE average $\overline{\sum_{\mu \neq \lambda} 1 /\left(E_{\lambda}-E_{\mu}\right)^{4}}$. If we introduce a small level spacing cutoff $\delta$, this average behaves as $1 / \delta$. Using the same regularization as in Section IV.B, i.e. $\delta \propto \Delta \bar{x}^{\eta / 2}$, we find a perturbative term of order $\Delta x^{3 \eta / 2}$. Carrying our regularization procedure to higher order terms we make the following conjecture regarding the analytical behavior of $(\overline{77})$ for finite $\Delta \bar{x}$. For the GOP

$$
\frac{\overline{\left(\Delta \Gamma_{\lambda}\right)^{2}}}{4 \bar{\Gamma}_{\lambda}^{2}}=|\Delta \bar{x}|^{\eta}\left[h_{1}(\Delta \bar{x})-h_{2}(\Delta \bar{x}) \ln |\Delta \bar{x}|^{\eta}\right] \text {, }
$$

where $h_{1}$ and $h_{2}$ have power expansion in $(\Delta \bar{x})^{\eta}$. For the GUP

$$
\frac{\overline{\left(\Delta \Gamma_{\lambda}\right)^{2}}}{4 \bar{\Gamma}_{\lambda}^{2}}=|\Delta \bar{x}|^{\eta}\left[h_{1}(\Delta \bar{x})-h_{2}(\Delta \bar{x})|\Delta \bar{x}|^{\eta / 2}\right],
$$

where $h_{1}$ and $h_{2}$ also have power expansion in $(\Delta \bar{x})^{\eta}$ (but are different from the corresponding functions for the GOP). Our conjecture is supported by the exact results for the $N=2$ GP

derived in Section VII. Note that for small $\Delta \bar{x}$ the functions in brackets in (80) and (81) are just $\tilde{R} / D$.

\section{E. Semiclassical Determination of the Rotation Scaling and the Correlation Field}

To obtain a universal behavior it is necessary (for $\eta=2$ ) to scale the external parameter by $\sqrt{D}$ or alternatively by $\sqrt{R}$. These scaling factors are system-dependent and in principle could be determined semiclassically. In this section we present a nonrigorous yet simple semiclassical derivation of the rotation scaling factor $R$. The WKB wavefunction inside the dot is given by $\Psi \sim A e^{i S / \hbar}$, where $S$ is the classical action. In the limit $\hbar \rightarrow 0$, the sensitivity of $\Psi$ to $x$ is due to the $x$-dependence of the action and we find, from (58) 


$$
R \sim \frac{1}{2} \overline{\left.\frac{1}{\hbar} \frac{\partial S}{\partial x}\right|^{2}}
$$

In the important case where $x$ is a magnetic field $B$, it is possible to calculate (82) explicitly. In particular we would like to derive an expression for the correlation field $B_{c}$ defined by $\bar{B}_{r}=\sqrt{R} B \equiv B / B_{c}$. The change in the action when the field is changed by $\Delta B$ is given by

$$
\Delta S=\frac{e}{c} \int \Delta \mathbf{A} \cdot d \ell
$$

In the following we shall distinguish between open and closed dots. For open dots we write $\Delta S=\frac{e}{c} \frac{\Theta}{2 \pi} \Delta B$, where $\Theta$ is the area (times $2 \pi$ ) swept by a classical trajectory. Using (82) we then find

$$
R \sim \overline{\Theta^{2}} / 2 \Phi_{0}^{2}
$$

where $\Phi_{0}=h c / e$ is the flux unit. In a ballistic chaotic open dot the area distribution is given by $P(\Theta) \propto e^{-\alpha_{c l}|\Theta|}$ [8] so that $\overline{\Theta^{2}}=2 / \alpha_{c l}^{2}$ and $R=1 / \alpha_{c l}^{2} \phi_{0}^{2}$. The correlation field is then given by

$$
B_{c}=\alpha_{c l} \Phi_{0}
$$

We note that this is exactly the correlation field that appears in the semiclassical derivation of the conductance correlator in ballistic open dots [8]. We also note that $\alpha_{c l} \propto \tau_{\text {esc }}^{-1 / 2}$ where $\tau_{\text {esc }}$ is the mean escape time from the dot 38.

We now turn to the case of closed dots (the Coulomb blockade regime) which is the one relevant to this paper. For such dots the electron does not escape but continues to accumulate phase until time $\tau=h / \Delta$, where $\Delta$ is the mean level spacing [27]. Since $\Gamma \ll \Delta$, the decay time is much longer than $\tau$, and it is that time scale $\tau$ that determines the correlation field. The action difference $\Delta S$ is then calculated for this time $\tau$. We write

$$
\Delta S=\frac{e}{c} \int_{0}^{\tau} d t \varphi(t)
$$

where $\varphi(t) \equiv \Delta \mathbf{A}(r(t)) \cdot \mathbf{v}(t)$. To calculate $\overline{(\Delta S)^{2}}$ we follow the method used in 39

$$
\overline{(\Delta S)^{2}}=2 \frac{e^{2}}{c^{2}} \int_{0}^{\tau} d t \int_{0}^{\tau-t} d t^{\prime} \overline{\varphi(t) \varphi\left(t+t^{\prime}\right)} \approx 2 \frac{e^{2}}{c^{2}} \tau \int_{0}^{\infty} d t \overline{\varphi(0) \varphi(t)}
$$

Since the magnitude of the velocity $v$ along the orbit is constant, the length $\ell$ travelled in time $t$ is given by $\ell=v t$. Measuring $\varphi$ as a function of $\ell$ and for an electron with unit velocity $(v=1)$, we have $\int_{0}^{\infty} d t \overline{\varphi(0) \varphi(t)}=v \int_{0}^{\infty} d \ell \overline{\hat{\varphi}(0) \hat{\varphi}(\ell)}$, where $\hat{\varphi}(\ell)=\Delta \mathbf{A} \cdot \hat{\mathbf{v}}=\frac{1}{2} \Delta B d(\ell)$ with $d$ being the distance of the corresponding orbit segment from the origin. Defining a dimensionless geometrical constant $\alpha_{g}$ [39]

$$
\alpha_{g}=\mathcal{A}^{-3 / 2} \int_{0}^{\infty} d \ell \overline{d(0) d(\ell)}
$$

we find from (82) 


$$
R(\Delta B)^{2}=\alpha_{g} \pi^{2} \tau v \mathcal{A}^{-1 / 2}\left(\frac{\Phi}{\Phi_{0}}\right)^{2} .
$$

If we denote by $\tau_{c r} \equiv \mathcal{A}^{1 / 2} / v$ the time for the ballistic electron at the Fermi energy to cross the dot, then the correlation field can be written as 20

$$
B_{c}=\frac{1}{\pi \sqrt{\alpha_{g}}} \sqrt{\frac{\tau_{c r}}{\tau}} \frac{\Phi_{0}}{\mathcal{A}},
$$

where

$$
\frac{\tau}{\tau_{c r}}=\frac{\sqrt{2 m E \mathcal{A}}}{\hbar},
$$

and we have used $\Delta=2 \pi \hbar^{2} / m \mathcal{A}$. We note that our result (90) is similar to the one obtained for the field that is required to break time-reversal symmetry [40,27,39]. From (90) and (91) we find that $B_{c} \mathcal{A} / \Phi_{0} \propto \mathcal{N}^{-1 / 4}$ where $\mathcal{N}$ is the number of electrons in the dot. In the diffusive regime we expect the correlation field to be given by a similar expression except that the ballistic crossing time is replaced by the time it takes the electron to diffuse across the dot $\tau_{c r}=\frac{\pi}{2} h / E_{c}$ where $E_{c}$ is the Thouless energy. We then find

$$
B_{c} \propto \sqrt{\frac{\Delta}{E_{c}}} \frac{\Phi_{0}}{\mathcal{A}},
$$

in agreement with the fluctuation correlation field obtained in the supersymmetry method [18], and with the field strength that is required to break time reversal symmetry in disordered systems [41,42].

Finally we remark that for open dots the semiclassical conductance correlator [8] can be written in terms of the scaled field $\bar{B}_{r}$ as $c_{g}=\left(1+\Delta \bar{B}_{r}^{2}\right)^{-2}$. For open dots we find a similar form $c_{g}=\left(1+\Delta \bar{B}_{r}^{2} / \alpha_{2 r}^{2}\right)^{-2}$ (see Eq. (32) for $\beta=2$ ) but with $\alpha_{2 r} \approx 1.15$ (where we have converted $\alpha_{2}$ of Section III.B to its value $\alpha_{2 r}$ in the rotation scaling).

\section{UNIVERSAL DISTRIBUTIONS OF THE WIDTH VELOCITY}

In this section we restrict the discussion to the differentiable parametric dependence $(\eta=2)$ where the factors associated with the level velocity scaling and the rotation scaling are related to the second moment of the level velocity and width velocity, respectively. One may be interested in higher moments of these quantities or in general in their distribution. In fact, for any finite $\Delta x$ we can define an average velocity of the unfolded levels and widths with respect to the scaled parameter $\bar{x}$

$$
v \equiv \frac{\Delta \varepsilon}{\Delta \bar{x}} ; \quad r \equiv \frac{1}{\bar{\Gamma}} \frac{\Delta \Gamma}{\Delta \bar{x}} .
$$

Their respective distributions $P_{\Delta \bar{x}}(v)$ and $P_{\Delta \bar{x}}(r)$ are symmetric and universal for any given $\Delta \bar{x}$. We now apply the perturbation techniques of Section IV to derive expressions for these distributions in the limit of small $\Delta \bar{x}$. 
We first address the simpler case of the level velocity distribution $P(v)=$ $\overline{\delta(v-\Delta \varepsilon / \Delta \bar{x})}=(2 \pi)^{-1} \int_{-\infty}^{\infty} e^{i v t} \overline{e^{-i t \Delta \varepsilon / \Delta \bar{x}}}$. In first order perturbation theory $v=\left(H_{\lambda \lambda}^{\prime}-\right.$ $\left.H_{\lambda \lambda}\right) / \Delta \bar{x}$, and the conditional average on $H\left(x^{\prime}\right)$ at fixed $H(x)$ can be easily done by noticing that $H_{\lambda \lambda}^{\prime}-f H_{\lambda \lambda}$ is a Gaussian variable with zero mean. Since cummulants higher than second vanish for a Gaussian variable, we find

$$
\left.\overline{e^{-i t \Delta \varepsilon / \Delta \bar{x}}}\right|_{\text {fixed } H(x)}=e^{-\frac{1}{2} t^{2} \overline{(\Delta \varepsilon / \Delta \bar{x})^{2}}}=e^{-\frac{1}{2} t^{2}},
$$

where we have used $\overline{(\Delta \varepsilon / \Delta \bar{x})^{2}}=1+\mathcal{O}\left(\Delta \bar{x}^{2}\right)$ at fixed $H(x)$. Since the expression found in (94) is independent of $H(x)$, the average over the latter does not change it and

$$
P(v)=(2 \pi)^{-1 / 2} e^{-\frac{1}{2} v^{2}} .
$$

Thus in the limit of small $\Delta \bar{x}$, the level velocity distribution is simply a Gaussian. In Fig. (top panel) we show (for both the orthogonal and unitary cases) the universal distribution (95) (solid lines) and compare it with $P_{\Delta \bar{x}}(v)$ for $\Delta \bar{x}=0.1$ (circles), as calculated from the GP. Only at larger values $\Delta \bar{x}>0.2$ we observe deviations from the limiting distribution (95). In Fig. 0 (top panel) we show the universal form of the distributions $P_{\Delta \bar{x}}(v)$ for such larger values of $\Delta \bar{x}$.

We now turn to the more interesting calculation of $P_{\Delta \bar{x}}(r)$ in the limit of small $\Delta \bar{x}$. To leading order in perturbation theory (see (70) $) r=(\Delta \bar{x} \bar{\Gamma})^{-1} \sum_{\mu \neq \lambda}\left(\frac{H_{\mu \lambda}^{\prime}}{E_{\lambda}-E_{\mu}} \gamma_{\lambda}^{*} \gamma_{\mu}+\right.$ c.c.) is a Gaussian variable at fixed $H(x)$ with zero conditional mean. Therefore

$$
\left.\overline{e^{-i t \bar{\Gamma}^{-1} \Delta \Gamma / \Delta \bar{x}}}\right|_{\text {fixed } H(x)}=e^{-\frac{1}{2} t^{2} \bar{\Gamma}^{-2} \overline{(\Delta \Gamma / \Delta \bar{x})^{2}}},
$$

where the conditional average of the squared width's rate of change is

$$
\left.\frac{1}{\bar{\Gamma}^{2}} \overline{\left(\frac{\Delta \Gamma}{\Delta \bar{x}}\right)^{2}}\right|_{\text {fixed } H(x)}=2 \sum_{\mu \neq \lambda} \frac{\Gamma_{\mu} \Gamma_{\lambda} / \bar{\Gamma}^{2}}{\left(\varepsilon_{\mu}-\varepsilon_{\lambda}\right)^{2}} .
$$

Taking the Fourier transform of (96), we can express $P(r)$ as a GE average over Gaussian distributions

$$
P(r)=\frac{1}{2\left[\pi \sum_{\mu \neq \lambda} \frac{\Gamma_{\mu} \Gamma_{\lambda} / \bar{\Gamma}^{2}}{\left(\varepsilon_{\mu}-\varepsilon_{\lambda}\right)^{2}}\right]^{-1 / 2} e^{-\left[4 \sum_{\mu \neq \lambda} \frac{\Gamma_{\mu} \Gamma_{\lambda} / \bar{\Gamma}^{2}}{\left(\varepsilon_{\mu}-\varepsilon_{\lambda}\right)^{2}}\right]^{-1} r^{2}} .}
$$

Thus we have reduced the process average in the original expression for $P(r)$ to an ensemble average. Fig. (1 (bottom panel) shows this limiting distribution (98) by a solid line, while the distribution $P_{\Delta \bar{x}}(r)$ for $\Delta \bar{x}=0.1$ is shown by circles. Notice that the variance of the limiting distribution $P(r)$ is just $R / D=\pi^{2} / 3$ in the unitary case and $\tilde{R} / D$ in the orthogonal case. In Fig. 5 (bottom panel) we show the universal distributions $P_{\Delta \bar{x}}(r)$ for larger values of $\Delta \bar{x}$. We use a logarithmic scale in order to show more clearly the deviations ar larger values of $r$ from the limiting form (98).

We observe from Fig. 5 that for both the level and width velocity distributions the deviation from the corresponding limiting form occurs faster (with increasing $\Delta \bar{x}$ ) for the GUP than for the GOP. Also in each symmetry class (i.e. orthogonal or unitary) the change in the distribution as $\Delta \bar{x}$ increases is slower for the width velocity distribution than it is for the level velocity distribution. 


\section{CONDUCTANCE CORRELATIONS IN ASYMMETRIC DOTS}

In present experiments in the Coulomb blockade regime the dots do not have reflection symmetry. In this section we calculate the conductance correlator and its analytic shortdistance behavior for such asymmetric dots. We also express the rotation scaling in terms of RMS conductance velocity.

\section{A. Universal Correlators}

For dots without reflection symmetry $\Gamma_{c}^{l} \neq \Gamma_{c}^{r}$, and Eq. (9) should be used for the conductance $G$. If in addition the leads are asymmetric we need in general two different channel correlation matrices $M_{c c^{\prime}}^{l}, M_{c c^{\prime}}^{r}$ of dimensions $\Lambda^{l}, \Lambda^{r}$ to characterize the dot, assuming that channels at different leads are uncorrelated. However, Eq. (24) still holds for $\Gamma^{l, r}$ separately and the conductance correlator $c_{G}\left(x-x^{\prime}\right)$ depends only on the eigenvalues $\left(w_{c}^{2}\right)^{l, r}$ of $M_{c c^{\prime}}^{l, r}$ as in the symmetric case, since the invariance of the GP under global unitary transformations implies that the choice of $\hat{\phi}_{c}^{l, r}$ does not affect averages involving $\left\langle\hat{\phi}_{c}^{l, r} \mid \psi\right\rangle$.

We first investigate $c_{G}\left(x-x^{\prime}\right)$ for an asymmetric dot with one-channel leads. This is the case which is relevant to present experiments in the Coulomb blockade regime where one channel dominates in each lead [20]. The strongest deviation from the symmetric dot correlator is found for symmetric leads $\overline{\Gamma^{l}}=\overline{\Gamma^{r}}$. The correlator for this case is calculated from simulations of the GP (31) and presented in the top panel of Fig. 6 (dashed) and is compared with the symmetric dot (32) (dotted). As in Section III.B we can also fit this correlator to (32) but now we find $\alpha_{1}=0.37 \pm 0.04$ for the case of conserved time reversal symmetry (GOP) and $\alpha_{2}=0.54 \pm 0.04$ for broken time-reversal symmetry (GUP). Also shown in Fig. 6 are results from the Anderson model as described in Section III.B. In the top right panel (broken time-reversal symmetry), the parameter dependence is introduced by folding the cylinder into a torus and applying an additional magnetic field $B^{\prime}$ perpendicular

to the torus plane. This amounts to setting $\theta_{\alpha \beta}=\frac{2 \pi}{n_{x}} \Phi^{\prime} / \Phi_{0} \equiv \theta$ in (B3) if $\vec{r}_{\alpha}, \vec{r}_{\beta}$ are neighbors along $x$ and using $\theta$ as the parameter. In the case of highly asymmetric leads, the small width can be neglected in the denominator of Eq. (9), and $c_{G}$ reduces again to the width correlator. We conclude that the conductance correlator for an asymmetric dot with singlechannel leads varies between the two limiting cases of symmetric leads and a symmetric dot. We note that this variation is smaller in the unitary case. We also find that it takes a large asymmetry of the leads to see significant changes in the correlator. To study the effect of increased number of channels on the conductance correlator, we computed $c_{G}\left(x-x^{\prime}\right)$ for an asymmetric dot with multi-channels symmetric leads $\left(M^{l}=M^{r}\right)$ assuming uncorrelated and equivalent channels. The GP results for 8 channels leads and the Anderson model results with $4 \times 4$ leads are presented in Fig. 6 (bottom) and show $c_{G}\left(x-x^{\prime}\right)$ approaching the symmetric dot result. We emphasize that in this case the Anderson model results are not expected to agree with the GP since their channel correlation matrices differ, thus the agreement we find numerically suggests that the correlator in the asymmetric case converges to a limiting form as the number of channels increases and that this limit is the symmetric dot correlator. 


\section{B. Perturbative Expression for the Conductance Correlator}

Using the techniques of Section IV.C, we can calculate $c_{G}\left(x-x^{\prime}\right)$ perturbatively (to leading order in $\Delta x$ ) for an asymmetric dot with arbitrary leads. We use the notation $G=\left(e^{2} / h\right)(\pi / 2 k T) g_{\lambda}$ where $g_{\lambda} \equiv \Gamma_{\lambda}^{l} \Gamma_{\lambda}^{r} /\left(\Gamma_{\lambda}^{l}+\Gamma_{\lambda}^{r}\right)$ and define $\Delta g_{\lambda} \equiv g_{\lambda}\left(x^{\prime}\right)-g_{\lambda}(x)$. For a small change in $x$ we have

$$
\Delta g_{\lambda}=\left(\frac{\Gamma_{\lambda}^{l}}{\Gamma_{\lambda}}\right)^{2} \Delta \Gamma_{\lambda}^{r}+\left(\frac{\Gamma_{\lambda}^{r}}{\Gamma_{\lambda}}\right)^{2} \Delta \Gamma_{\lambda}^{l},
$$

where $\Gamma_{\lambda} \equiv \Gamma_{\lambda}^{l}+\Gamma_{\lambda}^{r}$ is the total width of the level $\lambda$. Since the two leads are uncorrelated we get

$$
\overline{\left(\Delta g_{\lambda}\right)^{2}}=\overline{\left(\frac{\Gamma_{\lambda}^{l}}{\Gamma_{\lambda}}\right)^{4}\left(\Delta \Gamma_{\lambda}^{r}\right)^{2}}+\overline{\left(\frac{\Gamma_{\lambda}^{r}}{\Gamma_{\lambda}}\right)^{4}\left(\Delta \Gamma_{\lambda}^{l}\right)^{2}} .
$$

Using the same methods of Section III.B, and taking advantage of the invariance of $\Gamma_{\lambda}^{l, r} / \Gamma_{\lambda}$ under an $x$-independent orthogonal (unitary) transformation, we find

$$
\begin{array}{r}
\overline{\left(\frac{\Gamma_{\lambda}^{l, r}}{\Gamma_{\lambda}}\right)^{4}\left(\Delta \Gamma_{\lambda}^{r, l}\right)^{2}}=N^{2}\left(\sum_{c} w_{c}^{4}-\frac{1}{N-1} \sum_{c \neq c^{\prime}} w_{c}^{2} w_{c^{\prime}}^{2}\right) \overline{\left(\frac{\Gamma_{\lambda}^{l, r}}{\Gamma_{\lambda}}\right)^{4}\left(\Delta \hat{\Gamma}_{1 \lambda}^{r, l}\right)^{2}} \\
\approx \operatorname{Tr}\left(M^{l, r}\right)^{2} \overline{\left(\frac{\Gamma_{\lambda}^{l, r}}{\Gamma_{\lambda}}\right)^{4}\left(\Delta \hat{\Gamma}_{1 \lambda}^{r, l}\right)^{2}}
\end{array}
$$

where $\hat{\Gamma}_{1 \lambda}^{r l l}$ is the partial decay width to a single normalized channel in the corresponding lead and the last equality is valid in the limit of large $N$.

To calculate the expectation values in (101) we first perform the conditional average over $H\left(x^{\prime}\right)$ (keeping $H(x)$ fixed), for which we can use relation (72) to obtain

$$
\overline{\left(\Delta g_{\lambda}\right)^{2}}=4 \tilde{R}\left[\operatorname{Tr}\left(M^{r}\right)^{2} \overline{\left(\frac{\Gamma_{\lambda}^{l}}{\Gamma_{\lambda}}\right)^{4} \hat{\Gamma}_{1 \lambda}^{r} \hat{\Gamma}_{1 \mu}^{r}}+\operatorname{Tr}\left(M^{l}\right)^{2} \overline{\left(\frac{\Gamma_{\lambda}^{r}}{\Gamma_{\lambda}}\right)^{4} \hat{\Gamma}_{1 \lambda}^{l} \hat{\Gamma}_{1 \mu}^{l}}\right],
$$

where $\mu$ denotes a level different from $\lambda$. Using the relation $c_{G}\left(x-x^{\prime}\right)=1-\overline{\left(\Delta g_{\lambda}\right)^{2}} / 2\left(\overline{g_{\lambda}^{2}}-\bar{g}_{\lambda}^{2}\right)$, and the independence of the eigenfunctions $\lambda$ and $\mu$ (in the limit $N \rightarrow \infty$ ), we obtain

$$
c_{G}\left(x-x^{\prime}\right) \approx 1-b_{\beta} \frac{\tilde{R}}{D}\left|\bar{x}-\bar{x}^{\prime}\right|^{2},
$$

where the constant $b_{\beta}$ is given by

$$
b_{\beta}=2 \frac{\overline{g_{\lambda}^{4}\left[\operatorname{Tr}\left(M^{r}\right)^{2} \frac{\hat{\Gamma}_{1 \lambda}^{r}}{\left(\Gamma_{\lambda}^{r}\right)^{4}}+\operatorname{Tr}\left(M^{l}\right)^{2} \frac{\hat{\Gamma}_{1 \lambda}^{l}}{\left(\Gamma_{\lambda}^{l}\right)^{4}}\right]}}{N\left(\overline{g_{\lambda}^{2}}-\bar{g}_{\lambda}^{2}\right)} .
$$

We remark that the quantities averaged over in (104) are functions of the independent (normalized) partial widths $\hat{\Gamma}_{c \lambda}^{l, r}$ through $\Gamma_{\lambda}^{l, r}=N \sum_{c}\left(w_{c}^{l, r}\right)^{2} \hat{\Gamma}_{c \lambda}^{l, r}$, and that each partial width 
is distributed according to a $\chi^{2}$ distribution in $\beta$ degrees of freedom with the same mean $\bar{\Gamma}_{c \lambda}^{l, r}=1 / N$. Therefore the constant $b_{\beta}$ is completely determined by the eigenvalues $\left(w_{c}^{l, r}\right)^{2}$ and the number of channels in each lead.

In the important case of a dot with one channel in each lead and symmetric leads $\left(\bar{\Gamma}^{l}=\bar{\Gamma}^{r}\right)$ we find $b_{1}=7 / 4$ and $b_{2}=3$. It follows that for the GOP

$$
c_{G}\left(x-x^{\prime}\right) \approx 1-\frac{7}{4}\left(\bar{x}-\bar{x}^{\prime}\right)^{2}\left(-\frac{\pi^{2}}{6} \ln \left|\bar{x}-\bar{x}^{\prime}\right|+\text { Const. }\right),
$$

while for the GUP

$$
c_{G}\left(x-x^{\prime}\right) \approx 1-\pi^{2}\left(\bar{x}-\bar{x}^{\prime}\right)^{2} .
$$

\section{The Rotation Scaling as the RMS Conductance Velocity}

In asymmetric dots' experiments it is not possible to measure the width directly and it is therefore useful to express the rotation scaling directly in terms of the conductance velocity rather than the width velocity (see Section IV.D). This is accomplished through the perturbative expression (102)

$$
\frac{1}{\bar{G}_{\lambda}^{2}} \overline{\left(\Delta G_{\lambda}\right)^{2}} \approx 2 \tilde{R} b_{\beta} \frac{\overline{g^{2}}-\bar{g}^{2}}{\bar{g}^{2}}|\Delta x|^{2}
$$

Eq. (107) leads us to yet another definition of the rotation scaling

$$
\tilde{R}=r_{\beta} \frac{1}{\bar{G}_{\lambda}^{2}} \overline{\left(\frac{\Delta G_{\lambda}}{\Delta x}\right)^{2}}
$$

where $r_{\beta}=\left[2 b_{\beta}\left(\frac{\overline{g^{2}}-\bar{g}^{2}}{\bar{g}^{2}}\right)\right]^{-1}$.

In the case of one-channel symmetric leads, we find

$$
\tilde{R} \equiv \frac{1}{7} \frac{1}{\bar{G}_{\lambda}^{2}} \overline{\left(\frac{\Delta G_{\lambda}}{\Delta x}\right)^{2}}
$$

for the GOP, and

$$
R=\frac{5}{24} \frac{1}{\bar{G}_{\lambda}^{2}} \overline{\left(\frac{\partial G_{\lambda}}{\partial x}\right)^{2}}
$$

for the GUP.

In practice, one can calculate $R$ from the measured conductance peaks according to (109) or (110), and then use the universal ratio $R / D$, as given by Eq. (68) or Eq. (63), to determine the diffusion scaling factor $D$ for the GOP or GUP, respectively. The scaled parameter $\bar{x}$ that leads to universal correlations is now easily determined from (20). 


\section{THE $N=2$ GP}

For $N=2$ it is possible to calculate analytically the width correlator discussed in this paper. While the results are different from the $N \rightarrow \infty$ limit, it is nevertheless instructive to study the $N=2$ case. In particular the analytical properties of the rotation scaling factor and the width correlator are similar.

\section{A. Two-matrix distribution}

A GOE $2 \times 2$ matrix is real symmetric and thus has three independent real variables $H_{11}, H_{22}$ and $H_{12}$. We parametrize it in terms of its eigenvalues $E_{1}, E_{2}$ and the rotation angle $\alpha$ that characterizes the orthogonal $S O(2)$ matrix $R$ that diagonalizes $H$. Introducing the level spacing $E=E_{1}-E_{2}$ and average energy $\bar{E}=\left(E_{1}+E_{2}\right) / 2$, the GOE probability distribution is $\mathcal{P}_{1}(E, \bar{E}, \alpha) \propto|E| e^{-\bar{E}^{2} / a^{2}} e^{-E^{2} / 4 a^{2}}$, where $-\infty<E, \bar{E}<\infty$ and $-\pi / 2<\alpha<$ $\pi / 2$.

To solve for various two-point correlators, it is sufficient to calculate the joint two-matrix distribution $P\left(H, H^{\prime}\right)$ where $H \equiv H(x)$ and $H^{\prime} \equiv H\left(x^{\prime}\right)$. Introducing the quantities $E, \bar{E}, \alpha$ and $E^{\prime}, \bar{E}^{\prime}, \alpha^{\prime}$ for $H$ and $H^{\prime}$, respectively, we find

$$
\begin{aligned}
& P_{1}\left(E, E^{\prime}, \bar{E}, \bar{E}^{\prime}, \alpha, \chi\right)= \\
& \frac{\left|E E^{\prime}\right|}{4\left(\pi a^{2}\right)^{3}\left(1-f^{2}\right)^{3 / 2}} e^{-\left(\bar{E}^{2}+\bar{E}^{\prime 2}-2 f \bar{E} \bar{E}^{\prime}\right) / a^{2}\left(1-f^{2}\right)} e^{-\left(E^{2}+E^{\prime 2}-2 f \cos 2 \chi\right) / 4 a^{2}\left(1-f^{2}\right)},
\end{aligned}
$$

where $\chi=\alpha-\alpha^{\prime}$ is the rotation angle of $R^{-1} R^{\prime}$. Introducing polar coordinates for $E, E^{\prime}$ through $E=\zeta \cos \theta, E^{\prime}=\zeta \sin \theta$ and integrating over $\bar{E}, \bar{E}^{\prime}$ we obtain

$$
P_{1}(\zeta, \theta, \alpha, \chi)=\frac{1}{8\left(\pi a^{2}\right)^{2}\left(1-f^{2}\right)} \zeta^{3}|\sin 2 \theta| e^{-(1-f \sin 2 \theta \cos 2 \chi) \zeta^{2} / 4 a^{2}\left(1-f^{2}\right)},
$$

where $0<\zeta<\infty,-\pi<\theta<\pi$ and $-\pi / 2<\alpha, \chi<\pi / 2$.

For wavefunction correlators that are independent of the spectra, we can integrate over $\zeta$ and $\theta$. In order to keep the two levels at $x$ and $x^{\prime}$ in the same order we require $E E^{\prime}>0$, and therefore the integration range for $\theta$ is $0 \leq \theta \leq \pi / 2$ and $-\pi \leq \theta \leq-\pi / 2$. We obtain

$$
\begin{aligned}
& P_{1}(\alpha, \chi)=\frac{2\left(1-f^{2}\right)}{\pi^{2}} g_{1}(\xi) \\
& g_{1}(\xi)=\frac{1}{1-\xi^{2}}\left(1+\frac{2 \xi}{\sqrt{1-\xi^{2}}} \arctan \sqrt{\frac{1+\xi}{1-\xi}}\right) ; \quad \xi \equiv f \cos 2 \chi
\end{aligned}
$$

A $2 \times 2$ GUE matrix is characterized by two real variables $H_{11}, H_{22}$ and one complex variable $H_{12}$. We parametrized it by its eigenvalues $E_{1}, E_{2}$ and its diagonalizing unitary matrix

$$
U=\left(\begin{array}{cc}
e^{i \phi} \cos \alpha e^{i \psi} & e^{-i \phi} \sin \alpha e^{i \psi} \\
-e^{i \phi} \sin \alpha e^{-i \psi} & e^{-i \phi} \cos \alpha e^{-i \psi}
\end{array}\right)
$$


where $0 \leq \alpha \leq \pi, 0 \leq \phi \leq 2 \pi,-\pi \leq \psi \leq \pi$. We remark that the matrix $H$ is independent of $\psi$ but it is more convenient to use the parametrization (114) since it describes an element of the group $S U(2)$ whose invariant measure is $D[U]=|\sin 2 \alpha| d \alpha d \phi d \psi$. The GUE distribution is then given by $\mathcal{P}_{2}(E, \bar{E}, \alpha, \psi, \phi) \propto E^{2} \sin 2 \alpha e^{-2 \bar{E}^{2} / a^{2}} e^{-E^{2} / 2 a^{2}}$.

To parametrize the two-matrix distribution we introduce $E, \bar{E}, U$ and $E^{\prime}, \bar{E}^{\prime}, U^{\prime}$ to describe $H$ and $H^{\prime}$, respectively. Instead of $U$ and $U^{\prime}$ we then use $U$ and $\mathcal{U} \equiv U^{\dagger} U^{\prime}$. Parametrizing $\mathcal{U}$ as in (114) but with angles $\chi, \Phi, \Psi$ (replacing $\alpha, \phi, \psi)$ and using the invariance of the group measure $D\left[U^{\prime}\right]=D[\mathcal{U}]$, we find

$$
\begin{aligned}
& P_{2}\left(E, E^{\prime}, \bar{E}, \bar{E}^{\prime}, \alpha, \chi\right) \propto \\
& E^{2} E^{\prime 2}|\sin 2 \alpha \sin 2 \chi| e^{-2\left(\bar{E}^{2}+\bar{E}^{\prime 2}-2 f \bar{E} \bar{E}^{\prime}\right) / a^{2}\left(1-f^{2}\right)} e^{-\left(E^{2}+E^{\prime 2}-2 f E E^{\prime} \cos 2 \chi\right) / 2 a^{2}\left(1-f^{2}\right)} .
\end{aligned}
$$

Integrating over $\bar{E}, \bar{E}^{\prime}, \phi, \Phi$ and $\psi, \Psi$, and using polar coordinates $\zeta, \theta$ in the $E, E^{\prime}$ plane, we find

$$
P_{2}(\zeta, \theta, \alpha, \chi) \propto \zeta^{5} \sin ^{2} 2 \theta|\sin 2 \alpha \sin 2 \chi| e^{-\zeta^{2}(1-2 f \sin 2 \theta \cos 2 \chi) / 2 a^{2}\left(1-f^{2}\right)} .
$$

If $\zeta$ and $\theta$ are integrated out we have

$$
\begin{aligned}
& P_{2}(\alpha, \chi) \propto|\sin 2 \alpha \sin 2 \chi| g_{2}(\xi) \\
& g_{2}(\xi)=\frac{1}{\left(1-\xi^{2}\right)^{2}}\left(\frac{3}{2} \xi+\frac{2 \xi^{2}+1}{\sqrt{1-\xi^{2}}} \arctan \sqrt{\frac{1+\xi}{1-\xi}}\right) ; \quad \xi \equiv f \cos 2 \chi .
\end{aligned}
$$

\section{B. The Width Correlator}

We take a channel $\phi=\left(\begin{array}{l}1 \\ 0\end{array}\right)$ and the first eigenfunction $\psi(x)=\psi_{1}(x)$ so that for the GOP $\Gamma=R_{11}^{2}=\cos ^{2} \alpha$ and $\Gamma^{\prime}=R_{11}^{\prime 2}=\cos ^{2} \alpha^{\prime}=\cos ^{2}(\alpha+\chi)$. The correlation between $\Gamma$ and $\Gamma^{\prime}$ is then given by

$$
\overline{\Gamma \Gamma^{\prime}}=\overline{\cos ^{4} \alpha} \overline{\cos ^{2} \chi}+\frac{1}{4} \overline{\sin ^{2} 2 \alpha} \overline{\sin ^{2} \chi}-\frac{1}{2} \overline{\cos ^{2} \alpha \sin 2 \alpha} \overline{\sin 2 \chi}=\frac{1}{8}\left(1+2 \overline{\cos ^{2} \chi}\right),
$$

where we used $\overline{\cos ^{4} \alpha}=3 / 8$ and $\overline{\sin ^{2} 2 \alpha}=1 / 2$. Using $\overline{\Gamma^{2}}=3 / 8$, we obtain for the width correlator

$$
c_{\Gamma}=\overline{\cos 2 \chi}
$$

For the GUP $\Gamma=\left|U_{11}\right|^{2}=\cos ^{2} \alpha$ and $\Gamma_{1}^{\prime}=\left|U_{11}^{\prime}\right|^{2}=\cos ^{2} \alpha^{\prime}=\mid \cos \alpha \cos \chi-$ $\left.\sin \alpha \sin \chi e^{-2 i(\phi+\Psi)}\right|^{2}$, and therefore

$$
\begin{aligned}
\overline{\Gamma \Gamma^{\prime}} & =\overline{\cos ^{4} \alpha} \overline{\cos ^{2} \chi}+\frac{1}{4} \overline{\sin ^{2} 2 \alpha} \overline{\sin ^{2} \chi}-\frac{1}{2} \overline{\cos ^{2} \alpha \sin 2 \alpha} \overline{\operatorname{Re} e^{-2 i(\phi+\Psi)}} \overline{\sin 2 \chi} \\
& =\left(1+\overline{\cos ^{2} \chi}\right) / 6
\end{aligned}
$$

where we have used $\overline{\cos ^{4} \alpha}=1 / 3$ and $\overline{\sin ^{2} 2 \alpha}=2 / 3$. Since $\overline{\Gamma^{2}}=1 / 3$ we obtain again Eq. (119) for $c_{\Gamma}$. 
Eq. (119) can be calculated analytically for both classes. In the orthogonal case we have

$$
c_{\Gamma}^{\beta=1}=\frac{1-f^{2}}{4 \pi} \int_{0}^{\pi / 2} \cos 2 \chi\left[g_{1}(\xi)-g_{1}(-\xi)\right] d \chi=\frac{1-f^{2}}{4} \int_{0}^{\pi / 2} \frac{f \cos ^{2} 2 \chi}{\left(1-f^{2} \cos ^{2} 2 \chi\right)^{3 / 2}} d \chi .
$$

The integral in (121) can be evaluated to give

$$
c_{\Gamma}^{\beta=1}=\frac{\pi}{4} f\left(1-f^{2}\right)_{2} F_{1}\left(3 / 2,3 / 2 ; 2, f^{2}\right),
$$

where ${ }_{2} F_{1}$ is a hypergeometric function. In the unitary case we find

$$
\begin{aligned}
c_{\Gamma}^{\beta=2} & =\frac{\left(1-f^{2}\right)^{3 / 2}}{\pi} \int_{0}^{\pi / 2} \sin 2 \chi \cos 2 \chi\left[g_{2}(\xi)-g_{2}(-\xi)\right] d \chi \\
& =\left(1-f^{2}\right) \int_{0}^{\pi / 2} \frac{f \cos ^{2} 2 \chi}{\left(1-f^{2} \cos ^{2} 2 \chi\right)^{3 / 2}} d \chi .
\end{aligned}
$$

Here too the integral can be done exactly to give

$$
c_{\Gamma}^{\beta=2}=2 \frac{\sqrt{1-f^{2}}}{\pi f}+2 \frac{2 f^{2}-1}{\pi f^{2}} \arcsin f .
$$

\section{The width Correlator at Small Distances}

We now examine the behavior of $\overline{(\Delta \Gamma)^{2}} / 4 \overline{\Gamma^{2}}=\left(1-c_{\Gamma}\right) / 2(\beta+1)$ for small $\Delta \bar{x} \equiv \bar{x}-\bar{x}^{\prime}$ (see Eq. (77)) by expanding the exact solution for $c_{\Gamma}$ in $1-f \approx \beta \pi^{2}(\Delta \bar{x})^{2} / 8$.

For the GUP we find that (124) has a power expansion in $(1-f)^{1 / 2}$ and we obtain the general form (81) with $\eta=2$ suggested in Section IV.D where

$$
\begin{aligned}
& h_{1}(\Delta \bar{x})=\frac{\pi^{2}}{12}+\frac{\pi^{4}}{32}(\Delta \bar{x})^{2}+\ldots \\
& h_{2}(\Delta \bar{x})=\frac{\sqrt{2} \pi^{2}}{9}+\frac{29 \pi^{4}}{360 \sqrt{2}}(\Delta \bar{x})^{2}+\ldots .
\end{aligned}
$$

The leading order behavior agrees with the one derived from perturbation theory. We first use (63) where for $N=2$ the relevant $Z$ is Dyson's $Z_{D}=1 / 4$ (see Eq. (50)) to find $R / D=\pi^{2} / 8$. From (77) we then obtain

$$
\frac{\overline{(\Delta \Gamma)^{2}}}{4 \bar{\Gamma}^{2}} \approx \frac{\pi^{2}}{12}(\Delta \bar{x})^{2},
$$

in agreement with the leading term in (125).

For the GOP we find after expanding (122) the general from (80) where

$$
\begin{aligned}
& h_{1}(\Delta \bar{x})=\pi^{2} \frac{3 \ln 2-1-\ln \left(\pi^{2} / 8\right)}{64}+\mathcal{O}\left(\Delta \bar{x}^{2}\right) \\
& h_{2}(\bar{x})=\frac{\pi^{2}}{32}+\frac{3 \pi^{4}}{1024}(\Delta \bar{x})^{2}+\mathcal{O}\left(\Delta \bar{x}^{4}\right) .
\end{aligned}
$$


Thus the leading order terms in $h_{1}$ and $h_{2}$ are constants, which is consistent with the large $N$ perturbative result (68) for $\tilde{R} / D$ (although with different constants). It is instructive to repeat the perturbative GOP calculation for the special case $N=2$ and see whether our regularization procedure for this case yields the correct expansion. We obtain (77) with $\tilde{R} / D$ given by (62) but now

$$
Z=\frac{2}{\pi^{2}} \overline{\frac{1}{\left(\varepsilon_{1}-\varepsilon_{2}\right)^{2}}}=\frac{1}{4} \int_{\delta}^{\infty} \frac{1}{\varepsilon} e^{-\pi^{2} \varepsilon^{2} / 16} d \varepsilon=-\frac{1}{8} \operatorname{Ei}\left(-\frac{\pi^{2} \delta^{2}}{16}\right),
$$

where we introduce a small level spacing cutoff $\delta$ (in units of the mean spacing) and Ei is the exponential integral function. Using the identity $\operatorname{Ei}(-t)=\mathcal{C}+\ln t+\int_{0}^{t} d t^{\prime}\left(e^{-t^{\prime}}-1\right) / t^{\prime}$, where $\mathcal{C}$ is the Euler constant, we find

$$
Z=-\frac{1}{8}\left[\mathcal{C}+\ln \left(\frac{\pi^{2} \delta^{2}}{16}\right)+\mathcal{O}\left(\delta^{2}\right)\right]
$$

Making the assumption $\delta^{2} \propto(\Delta \bar{x})^{2}$, the quantity (17) is then given by

$$
\frac{\overline{(\Delta \Gamma)^{2}}}{4 \bar{\Gamma}^{2}}=(\Delta \bar{x})^{2}\left[-\frac{\pi^{2}}{32} \ln (\Delta \bar{x})^{2}+\text { Const. }\right] \text {, }
$$

in agreement with (81) and the leading order in (127).

\section{CONCLUSION}

In this paper we discussed the parametric correlation function $c_{G}\left(x-x^{\prime}\right)$ of the conductance peaks in quantum dots in the regime of Coulomb blockade oscillations. Using the framework of the Gaussian process, we demonstrated its universality and obtained its functional form as well as its exact analytic behavior at short distances for both cases of conserved and broken time-reversal symmetry. For a symmetric dot we proved that $c_{G}$ is independent of the channel configuration, while for asymmetric dots with multi-channel leads, our results indicate that the conductance correlator approaches its symmetric-dot form as the number of channels increases. We proposed a new scaling procedure, the rotation scaling, which uses a scaling factor that can be extracted directly from the conductance peaks and is therefore experimentally measurable. Our main result (32) can be readily tested experimentally. It would also be interesting to apply our rotation scaling to chaotic systems other than quantum dots.

This work was supported in part by the Department of Energy Grant DE-FG0291ER40608. We acknowledge C.M. Marcus for useful discussions.

\section{APPENDIX A: USEFUL GE RELATIONS}

In this appendix we derive various useful partial width amplitudes correlations in the Gaussian ensembles. In the following $\phi_{1}, \phi_{2}$ denote two orthogonal and normalized channels, while $\psi_{\lambda}, \psi_{\mu}$ are two eigenfunction $(\lambda \neq \mu) . \gamma_{c \lambda}=\left\langle\phi_{c} \mid \psi_{\lambda}\right\rangle$ are the partial width amplitudes. 
We first derive the distribution of the partial width amplitudes $\gamma_{1 \lambda}, \gamma_{2 \lambda}, \gamma_{1 \mu}, \gamma_{2 \mu}$, which is given by

$$
P\left(\gamma_{1 \lambda}, \gamma_{2 \lambda}, \gamma_{1 \mu}, \gamma_{2 \mu}\right)=\int \prod_{j=3}^{N}\left(d \psi_{j \lambda} d \psi_{j \mu}\right) \delta\left(\sum_{i=1}^{N}\left|\psi_{i \lambda}\right|^{2}-1\right) \delta\left(\sum_{i=1}^{N}\left|\psi_{i \mu}\right|^{2}-1\right) \delta\left(\sum_{i=1}^{N} \psi_{i \lambda}^{*} \psi_{i \mu}\right)
$$

Using two sets of spherical coordinates for $\psi_{j \lambda} ; j=3, \ldots, N$ and $\psi_{j \mu} ; j=3, \ldots, N$ in (A1) we find

$$
P(\gamma) \propto\left[\left(1-R_{\lambda}^{2}\right)\left(1-R_{\mu}^{2}\right)-|S|^{2}\right]^{\beta \frac{N-3}{2}-1}
$$

where $R_{\lambda}^{2}=\left|\gamma_{1 \lambda}\right|^{2}+\left|\gamma_{2 \lambda}\right|^{2}$ and $S=\gamma_{1 \lambda}^{*} \gamma_{1 \mu}+\gamma_{2 \lambda}^{*} \gamma_{2 \mu}$. From (A2) we can calculate various moments and correlations of the partial amplitudes.

However, most of the useful relations can be derived in a simpler way. From the exact distribution of a single amplitude [5], $P\left(\gamma_{1 \lambda}\right) \propto\left(1-\left|\gamma_{1 \lambda}\right|^{2}\right)^{\beta \frac{N-1}{2}-1}$, we find

$$
\overline{\left|\gamma_{1 \lambda}\right|^{4}}=\frac{1}{N} \frac{\beta+2}{\beta N+2}
$$

By squaring the relation $\sum_{i=1}^{N}\left|\gamma_{i \lambda}\right|^{2}=1$, taking an average and using (A3) we find

$$
\overline{\left|\gamma_{1 \lambda}\right|^{2}\left|\gamma_{2 \lambda}\right|^{2}}=\frac{1}{N} \frac{\beta}{\beta N+2} .
$$

Similarly, if we average the square the relation $\sum_{\lambda=1}^{N}\left|\gamma_{1 \lambda}\right|^{2}=1$ and use (A3) we obtain

$$
\overline{\left|\gamma_{1 \lambda}\right|^{2}\left|\gamma_{1 \mu}\right|^{2}}=\frac{1}{N} \frac{\beta}{\beta N+2}
$$

To derive the correlation between the widths of two eigenfunctions to decay into two orthogonal channels, we use $\sum_{i, j=1}^{N} \overline{\left|\gamma_{i \lambda}\right|^{2}\left|\gamma_{j \mu}\right|^{2}}=1$ together with (A5)

$$
\overline{\left|\gamma_{1 \lambda}\right|^{2}\left|\gamma_{2 \mu}\right|^{2}}=\frac{\beta(N-1)+2}{\beta N+2} \frac{1}{N(N-1)} .
$$

Finally, by averaging the square of the modulus of the orthogonality relation of the two eigenvectors $\sum_{i=1}^{N} \gamma_{i \lambda}^{*} \gamma_{i \mu}=0$, and using relation (A5), we find

$$
\overline{\gamma_{1 \lambda}^{*} \gamma_{1 \mu} \gamma_{2 \lambda} \gamma_{2 \mu}^{*}}=-\frac{1}{N-1} \overline{\left|\gamma_{1 \lambda}\right|^{2}\left|\gamma_{1 \mu}\right|^{2}}=-\frac{\beta}{\beta N+2} \frac{1}{N(N-1)}
$$




\section{APPENDIX B: PERTURBATIVE CALCULATION OF THE CROSS-CHANNEL AND EXCHANGE CORRELATORS}

To prove (27) to leading order in perturbation theory we consider the quantity $\overline{\left(\Gamma_{1 \lambda}^{\prime}-\Gamma_{1 \lambda}\right)\left(\Gamma_{2 \lambda}^{\prime}-\Gamma_{2 \lambda}\right)}$ where the subscripts 1,2 correspond to an orthogonal pair of normalized channel vectors $\phi_{1,2}$, and $\Gamma_{i \lambda}(x)=\left|\gamma_{i \lambda}(x)\right|^{2}=\left|\left\langle\phi_{i} \mid \psi_{\lambda}(x)\right\rangle\right|^{2}, i=1,2$. Using (56) we have

$$
\left(\Gamma_{1 \lambda}^{\prime}-\Gamma_{1 \lambda}\right)\left(\Gamma_{2 \lambda}^{\prime}-\Gamma_{2 \lambda}\right)=\left(\sum_{\mu \neq \lambda} \frac{H_{\mu \lambda}^{\prime}}{E_{\lambda}-E_{\mu}} \gamma_{\lambda}^{*} \gamma_{\mu}+c . c\right)\left(\sum_{\nu \neq \lambda} \frac{H_{\nu \lambda}^{\prime}}{E_{\lambda}-E_{\nu}} \gamma_{\lambda}^{*} \gamma_{\nu}+c . c\right)
$$

which upon averaging gives

$$
\overline{\left(\Gamma_{1 \lambda}^{\prime}-\Gamma_{1 \lambda}\right)\left(\Gamma_{2 \lambda}^{\prime}-\Gamma_{2 \lambda}\right)}=2\left(\overline{\gamma_{1 \lambda}^{*} \gamma_{2 \lambda} \gamma_{1 \mu} \gamma_{2 \mu}^{*}}+\text { c.c. }\right) R|\Delta x|^{\eta} \text {. }
$$

The cross-channel correlation is then calculated from (B2) using

$$
\overline{\Gamma_{1 \lambda} \Gamma_{2 \lambda}^{\prime}}-\overline{\Gamma_{1 \lambda}} \overline{\Gamma_{2 \lambda}^{\prime}}=\overline{\Gamma_{1 \lambda} \Gamma_{2 \lambda}}-\overline{\Gamma_{1 \lambda}} \overline{\Gamma_{2 \lambda}}-\overline{\left(\Gamma_{1 \lambda}^{\prime}-\Gamma_{1 \lambda}\right)\left(\Gamma_{2 \lambda}^{\prime}-\Gamma_{2 \lambda}\right)} / 2
$$

After also using the GE relation $\left(\overline{\gamma_{1 \lambda}^{*} \gamma_{2 \lambda} \gamma_{1 \mu} \gamma_{2 \mu}^{*}}+\right.$ c.c $) / 2=\frac{\beta}{2} \frac{N}{N-1}\left(\overline{\Gamma_{1 \lambda} \Gamma_{2 \lambda}}-\overline{\Gamma_{1 \lambda}} \overline{\Gamma_{2 \lambda}}\right)$ we get

$$
\overline{\Gamma_{1 \lambda} \Gamma_{2 \lambda}^{\prime}}-\overline{\Gamma_{1 \lambda}} \overline{\Gamma_{2 \lambda}^{\prime}}=\left(\overline{\Gamma_{1 \lambda} \Gamma_{2 \lambda}}-\overline{\Gamma_{1 \lambda}} \overline{\Gamma_{2 \lambda}}\right)\left(1-\beta \frac{N}{N-1} R|\Delta x|^{\eta}\right)
$$

Comparing with the autochannel correlator (74) and using the GE relation $\overline{\Gamma_{1} \Gamma_{2}}-\overline{\Gamma_{1}} \overline{\Gamma_{2}}=$ $-\frac{1}{N-1}\left(\overline{\Gamma_{1} \Gamma_{1}}-{\overline{\Gamma_{1}}}^{2}\right)$, we recover $(27)$ to order $|\Delta x|^{\eta}$.

Relation (38) can be similarly proven in perturbation theory. Writing the correlator of interest in the form $\left(\overline{\gamma_{1 \lambda}^{*} \gamma_{2 \lambda} \gamma_{1 \lambda}^{\prime *} \gamma_{2 \lambda}^{\prime}}+\right.$ c.c. $) / 2=\overline{\Gamma_{1 \lambda} \Gamma_{2 \lambda}}-\overline{\left|\gamma_{1 \lambda}^{*} \gamma_{2 \lambda}-\gamma_{1 \lambda}^{\prime *} \gamma_{2 \lambda}^{\prime}\right|^{2}} / 2$, we calculate the second term on the r.h.s. Here we have to distinguish between the GOP and the GUP. For the GOP

$$
\overline{\left|\gamma_{1 \lambda}^{*} \gamma_{2 \lambda}-\gamma_{1 \lambda}^{\prime *} \gamma_{2 \lambda}^{\prime}\right|^{2}}=2\left(\overline{\gamma_{1 \lambda}^{2} \gamma_{2 \mu}^{2}}+\overline{\gamma_{1 \lambda} \gamma_{2 \lambda} \gamma_{1 \mu} \gamma_{2 \mu}}\right) R|\Delta x|^{\eta}
$$

while for the GUP we find

$$
\overline{\left|\gamma_{1 \lambda}^{*} \gamma_{2 \lambda}-\gamma_{1 \lambda}^{\prime *} \gamma_{2 \lambda}^{\prime}\right|^{2}}=4 \overline{\left|\gamma_{1 \lambda}\right|^{2}\left|\gamma_{2 \mu}\right|^{2}} R|\Delta x|^{\eta}
$$

Using also the GOE relation $\overline{\Gamma_{1 \lambda} \Gamma_{2 \mu}}+\overline{\gamma_{1 \lambda} \gamma_{2 \lambda} \gamma_{1 \mu} \gamma_{2 \mu}}=\frac{N}{N-1} \overline{\Gamma_{1 \lambda} \Gamma_{2 \lambda}}$ and the GUE relation $\overline{\Gamma_{1 \lambda} \Gamma_{2 \mu}}=\frac{N}{N-1} \overline{\Gamma_{1 \lambda} \Gamma_{2 \lambda}}$, we obtain

$$
\frac{1}{2}\left(\overline{\gamma_{1 \lambda}^{*} \gamma_{2 \lambda} \gamma_{1 \lambda}^{* *} \gamma_{2 \lambda}^{\prime}}+\text { c.c. }\right)=\overline{\Gamma_{1 \lambda} \Gamma_{2 \lambda}}\left(1-\beta \frac{N}{N-1} R|\Delta x|^{\eta}\right)
$$

Comparing (B7) with ([4) and using $\overline{\Gamma_{1 \lambda} \Gamma_{2 \lambda}}=\frac{\beta}{2} \frac{N}{N-1}\left({\overline{\Gamma_{1 \lambda}^{2}}}_{-\bar{\Gamma}_{1 \lambda}}^{2}\right)$, we recover (38) to leading order in $|\Delta x|^{\eta}$. 


\section{REFERENCES}

[1] For a review see for example, M.A. Kastner, Rev. Mod. Phys. 64, 849 (1992); W.P. Kirk and M.A. Reed (Ed.), Nanostructures and Mesoscopic Systems (Academic Press, San Diego, 1992).

[2] U. Meirav, M.A. Kastner and S.J. Wind, Phys. Rev. Lett. 65, 771 (1990); P.L. McEuen et al. , Phys. Rev. Lett. 66, 1929 (1991); Physica B 189, 70 (1993); E.B. Foxman et al., Phys. Rev. B 47, 10020 (1993).

[3] C.W.J. Beenakker, Phys. Rev. B 44, 1646 (1991).

[4] R.A. Jalabert, A.D. Stone and Y. Alhassid, Phys. Rev. Lett. 68, 3468 (1992).

[5] M.L. Mehta, Random Matrices, 2nd ed. (Academic Press, New York, 1991); T.A. Brody, J. Flores, J.B. French, P.A. Mello, A. Pandey and S.S.M. Wong, Rev. Mod. Phys. 53, 385 (1981).

[6] O. Bohigas, in Chaos and Quantum Physics, ed. M.-J. Giannoni, A. Voros and J. ZinnJustin (North-Holland, Amsterdam, 1991).

[7] M.C. Gutzwiller, Chaos in Classical and Quantum Mechanics (Springer-Verlag, New York, 1990).

[8] R.A. Jalabert, H.U. Baranger and A.D. Stone, Phys. Rev. Lett. 65, 2442 (1990); H.U. Baranger, R.A. Jalabert and A.D. Stone, Chaos 3, 665 (1993).

[9] K.B. Efetov, Phys. Rev. Lett. 74, 2299 (1995).

[10] C.M. Marcus, R.M. Westervelt, P.F. Hopkins and A.C. Gossard, Phys. Rev B 48, 2460 (1993); Surf. Sci. 305, 480 (1994).

[11] I.H. Chan, R.M. Clarke, C.M. Marcus, K. Campman and A.C. Gossard, Phys. Rev. Lett. 74, 3876 (1995). 71, 1230 (1993).

[12] T. Ericson, Phys. Rev. Lett. 5, 430 (1960).

[13] C. E. Porter, Statistical Theory of Spectra: Fluctuations (Academic Press, New York, 1965).

[14] Y. Alhassid and H. Attias, Phys. Rev. Lett. 76, 1711 (1996).

[15] Y. Alhassid and H. Attias, Phys. Rev. Lett. 74, 4635 (1995).

[16] H. Attias and Y. Alhassid, Phys. Rev. E 52, 4476 (1995).

[17] M. Wilkinson, J. Phys. A 22, 2795 (1989).

[18] B.D. Simons and B.L. Altshuler, Phys. Rev. Lett. 70, 4063 (1993); Phys. Rev. B 48, 5422 (1993).

[19] B.D. Simons, P.A. Lee and B.L. Altshuler, Phys. Rev. Lett. 72, 64 (1994); Nucl. Phys. B 409, 487 (1993).

[20] J.A. Folk, S.R. Patel, S.F. Godijn, A.G. Huibers, S.M. Cronenwett, C.M. Marcus, K. Campman and A.C. Gossard, Phys. Rev. Lett. 76, 1699 (1996).

[21] A.M. Chang, H.U. Baranger, L.N. Pfeiffer, K.W. West and T.Y. Chang, Phys. Rev. Lett. 76, 1695 (1996).

[22] S. Sridhar, Phys. Rev. Lett. 67, 785 (1991); S. Sridhar, D. Hogenboom and B. Willemsen, J. Stat. Phys. 68, 239 (1992).

[23] H. Alt, H.-D. Gräf, H. L. Harney, R. Hofferbert, H. Lengeler, A. Richter, P. Schardt and H. A. Weidenmüller, Phys. Rev. Lett. 74, 62 (1995).

[24] A.M. Lane and R.G. Thomas, Rev. Mod. Phys. 30, 257 (1958).

[25] E.P. Wigner and L. Eisenbud, Phys. Rev. 72, 29 (1947). 
[26] J.M. Blatt and V.F. Weisskopf, Theoretical Nuclear Physics (Wiley, New York, 1952).

[27] A.D. Stone and H. Bruus, Physica B 189, 43 (1993): Surf. Sci. 305, 490 (1994).

[28] H. Bruus and A.D. Stone, Phys. Rev. B 50, 18275 (1994).

[29] R. Landauer, IBM J. Res. Dev. 1, 223 (1957); Philos. Mag. 21, 863 (1970); M. Büttiker, Phys. Rev. Lett. 57, 1761 (1986); IBM J. Res. Dev. 32, 317 (1988).

[30] E.R. Mucciolo, V.N. Prigodin and B.L. Altshuler, Phys. Rev. B 51, 1714 (1995).

[31] V.N. Prigodin, K.B. Efetov and S. Iida, Phys. Rev. Lett. 71, 1230 (1993).

[32] F.J. Dyson, J. Math. Phys. 3, 140, 157, 166, 1199 (1962).

[33] D. Mitchell, Y. Alhassid and D. Kusnezov, Yale preprint.

[34] E.A. Austin and M. Wilkinson, Nonlinearity 5, 1137 (1992).

[35] P.W. Anderson, Phys. Rev. 109, 1492 (1958).

[36] P.A. Lee and T.V. Ramakrishnan, Rev. Mod. Phys. 57, 287 (1985).

[37] F.J. Dyson, J. Math. Phys. 3, 1191 (1962).

[38] R.V. Jensen, Chaos 1, 101 (1991).

[39] O. Bohigas, M-J Giannoni, A.M Ozorio de Almeida and C. Schmit, Nonlinearity 8, 203 (1995).

[40] M.V. Berry and M. Robnik, J. Phys. A 19, 649 (1984).

[41] A. Altland, S. Iida and K.B. Efetov, J. Phys. A 26, 3545 (1993).

[42] V.I. Falko and K.B. Efetov, Phys. Rev. B 50, 11267 (1994). 


\section{FIGURES}

FIG. 1. Universal form of the conductance correlator for a symmetric dot (22). Shown are GP simulation results (diamonds) obtained from (31) and their best fit to (32) (dashed). The circles are results from the Anderson model in a cylindrical geometry for leads of $2 \times 2$ point contacts, and with the parameter being the strength of an external potential. In the case of broken time reversal symmetry (right) a magnetic flux of $\Phi / \Phi_{0}=1 / 4$ is applied. For comparison we also plot the GUP result on the left and the GOP on the right (dotted). The GP results are obtained using 300 simulations with $N=150$, taking the middle third of the states, while the Anderson model results are from the middle 200 eigenfunctions of the Hamiltonian (33) with $W=1$ on a $27 \times 27$ lattice.

FIG. 2. GP (diamonds with statistical error) and Anderson model (circles) simulation results for the scaling factors ratio $\tilde{R} / D$ computed with finite $\Delta x$, compared to the prediction in the orthogonal (68) and unitary (63) cases. Notice that the ratio is plotted as a function of $\ln \Delta \bar{x}$ for the orthogonal case and as a function of $\Delta \bar{x}$ for the unitary case. Here $\bar{x}=\sqrt{D} x$ is the diffusion scaling. The Anderson model results correspond to the cases shown in Fig. 11 but for $4 \times 4$ leads.

FIG. 3. The universal form of $\overline{(\Delta \Gamma)^{2}} / \bar{\Gamma}^{2}$ as a function of $(\Delta \bar{x})^{2}$ for the GOP (left) and the GUP (right). The results are obtained from simulations of the process (31). The asymptotic value for large $\Delta \bar{x}$ is $4 / \beta$.

FIG. 4. Top: the level velocity distribution $P(v=\partial \varepsilon / \partial \bar{x})$ given by (95) (solid line). Shown by circles is the distribution $P(v=\Delta \varepsilon / \Delta \bar{x})$ calculated for $\Delta \bar{x}=0.1$. Bottom: The width velocity distribution $P\left(r=\bar{\Gamma}^{-1} \partial \Gamma / \partial \bar{x}\right)$ given by (98) (calculated for $\Delta \bar{x}=0.01$ ). Also shown is the distribution $P\left(r=\bar{\Gamma}^{-1} \Delta \Gamma / \Delta \bar{x}\right)$ for $\Delta \bar{x}=0.1$ (circles).

FIG. 5. Top: the average level velocity distribution $P(v)$ (where $v=\Delta \varepsilon / \Delta \bar{x}$ ) for various values of $\Delta \bar{x}=0.25$ (squares), 0.5 (pluses), 0.75 (crosses) and 1 (diamonds). The solid line is the limiting Gaussian distribution (95). Bottom: the average width velocity distribution $P(r)$ (where $r=\bar{\Gamma}^{-1} \Delta \Gamma / \Delta \bar{x}$ ) for the same values of $\Delta \bar{x}$ as for $P(v)$. The solid histogram is the limiting distribution (98). Notice that we use a logarithmic scale for $P(r)$ in order to distinguish between the various cases at larger values of $r$.

FIG. 6. Universal form of the conductance correlator (11) in asymmetric dots. Top: GP simulation results with single-channel symmetric leads (dashed) and Anderson model results with $1 \times 1$ leads (pluses and crosses). Bottom: GP simulation results with 8 equivalent channels in each lead (dashed) and Anderson model results with $4 \times 4$ point-contact leads (squares and circles). The Anderson model is as described in Fig. 11 where the parametric dependence is on the strength of an external potential, except in one case (crosses) where the parametric dependence is that of an additional external magnetic field in a toroidal geometry. For comparison we also plot the correlator for a symmetric dot (32) (dotted). 


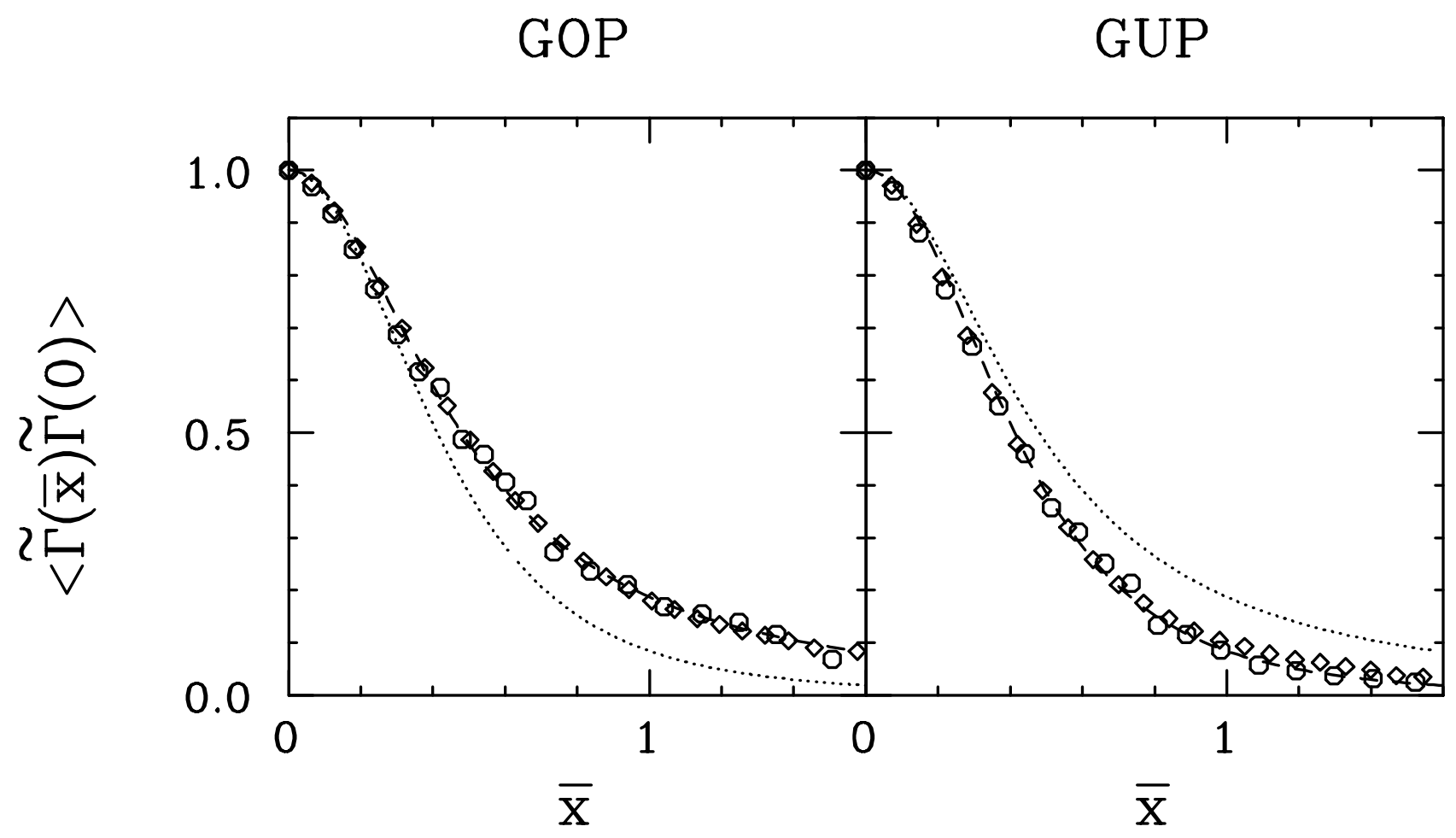




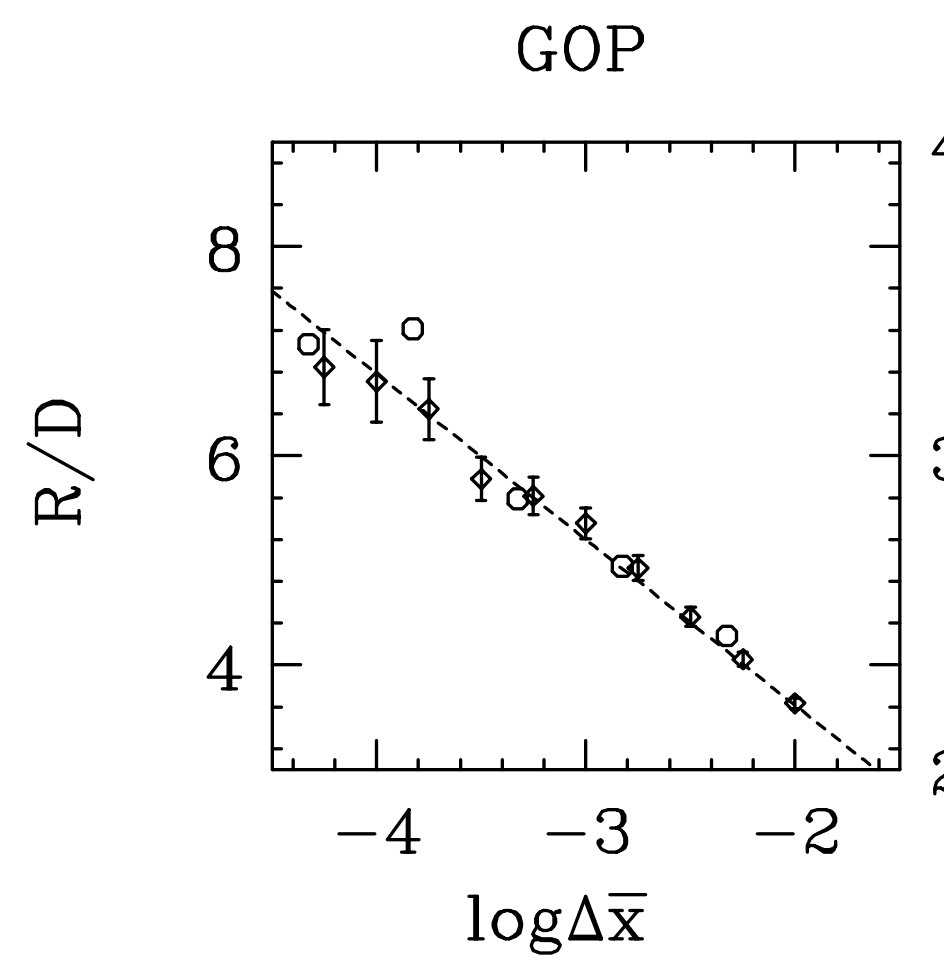

GUP

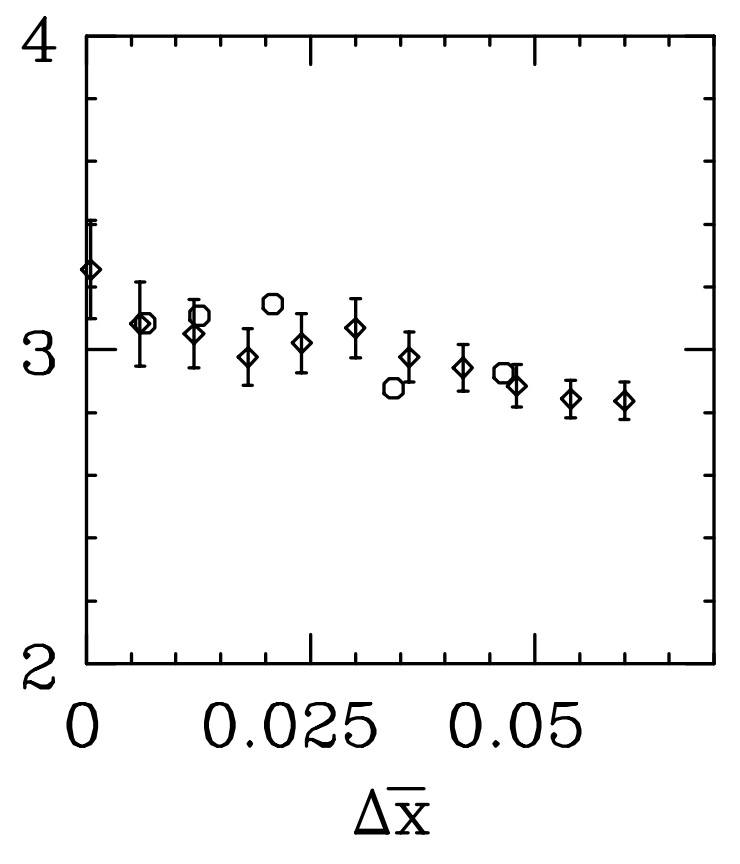




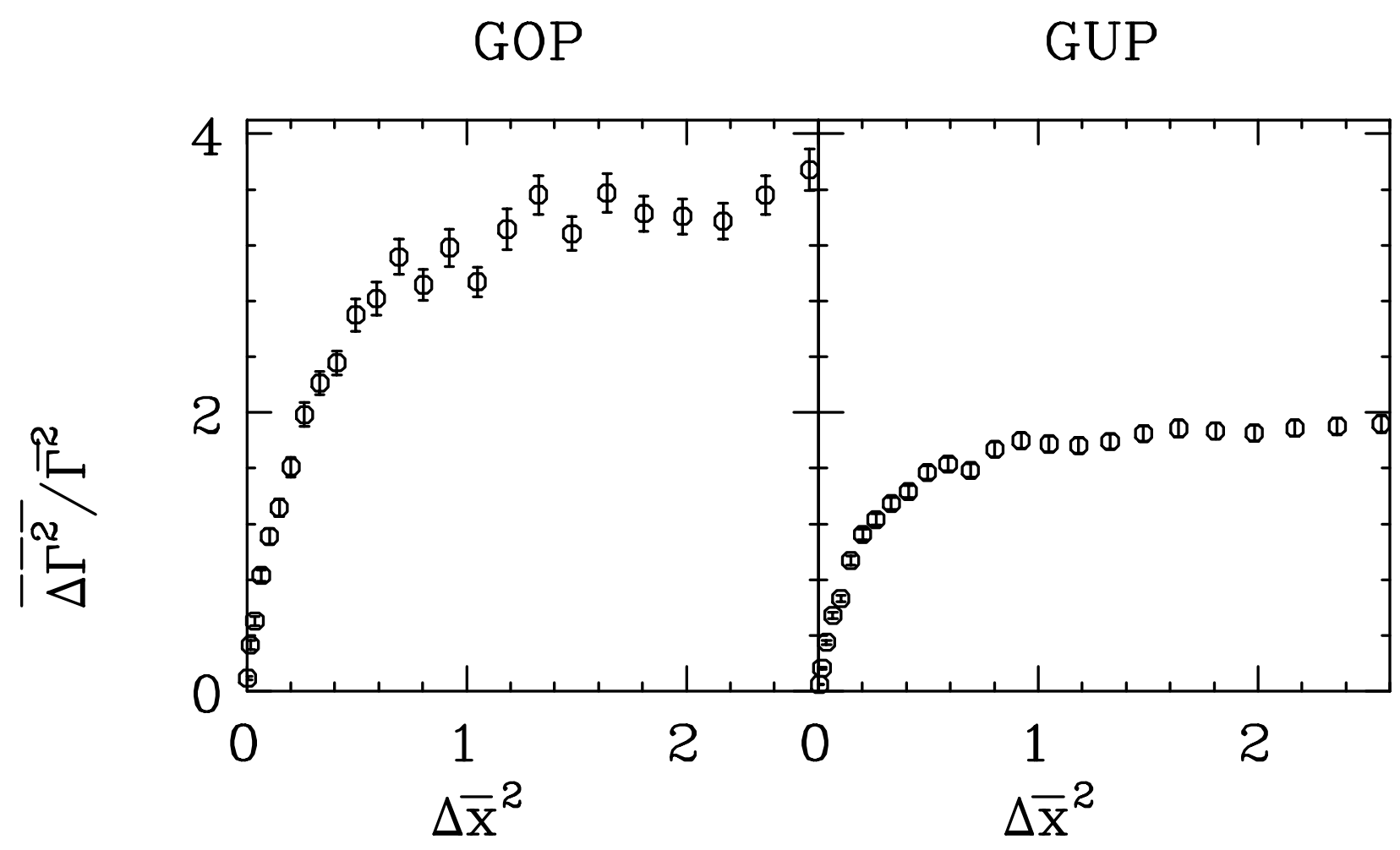


GOP

GUP
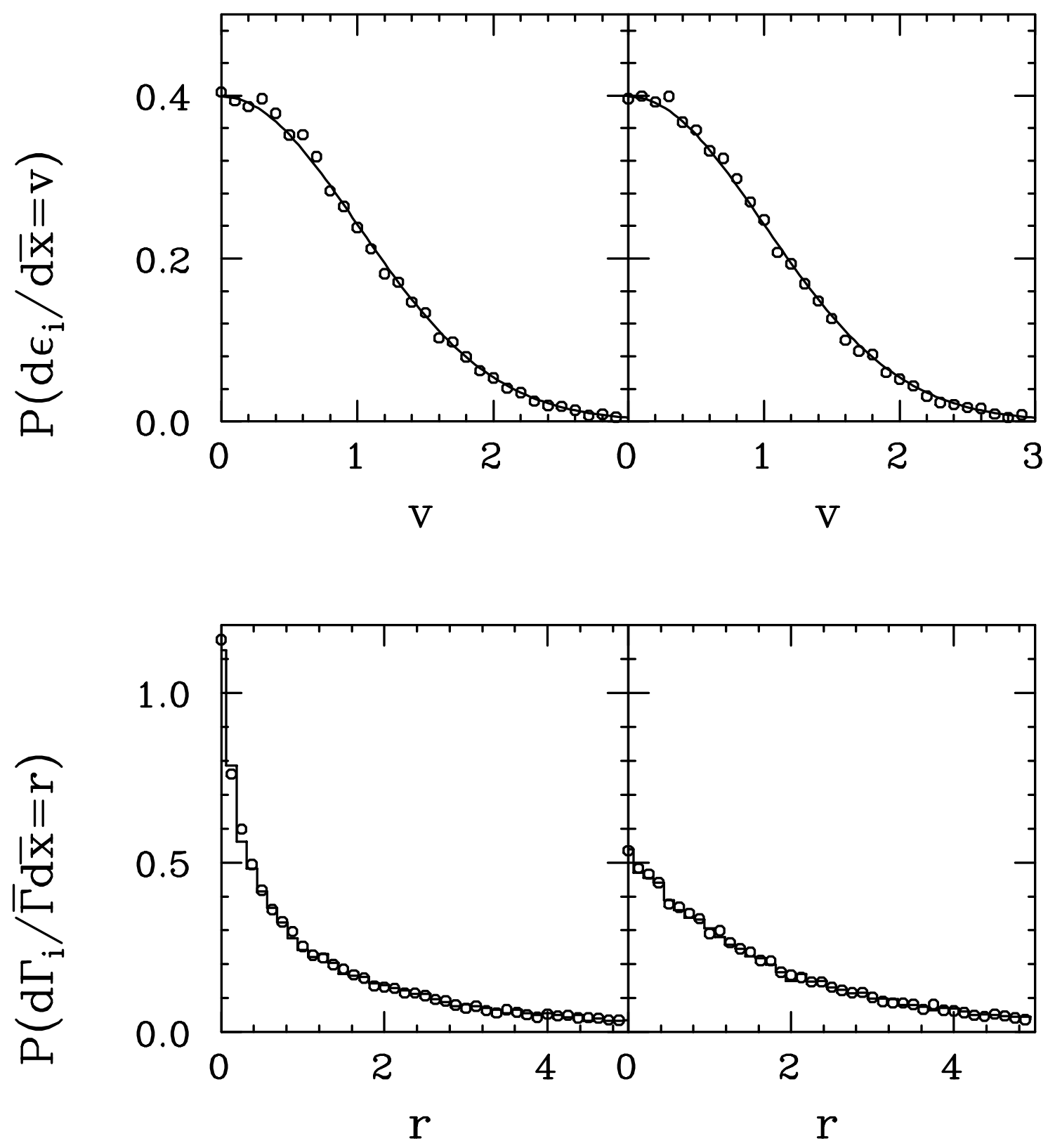
GOP GUP
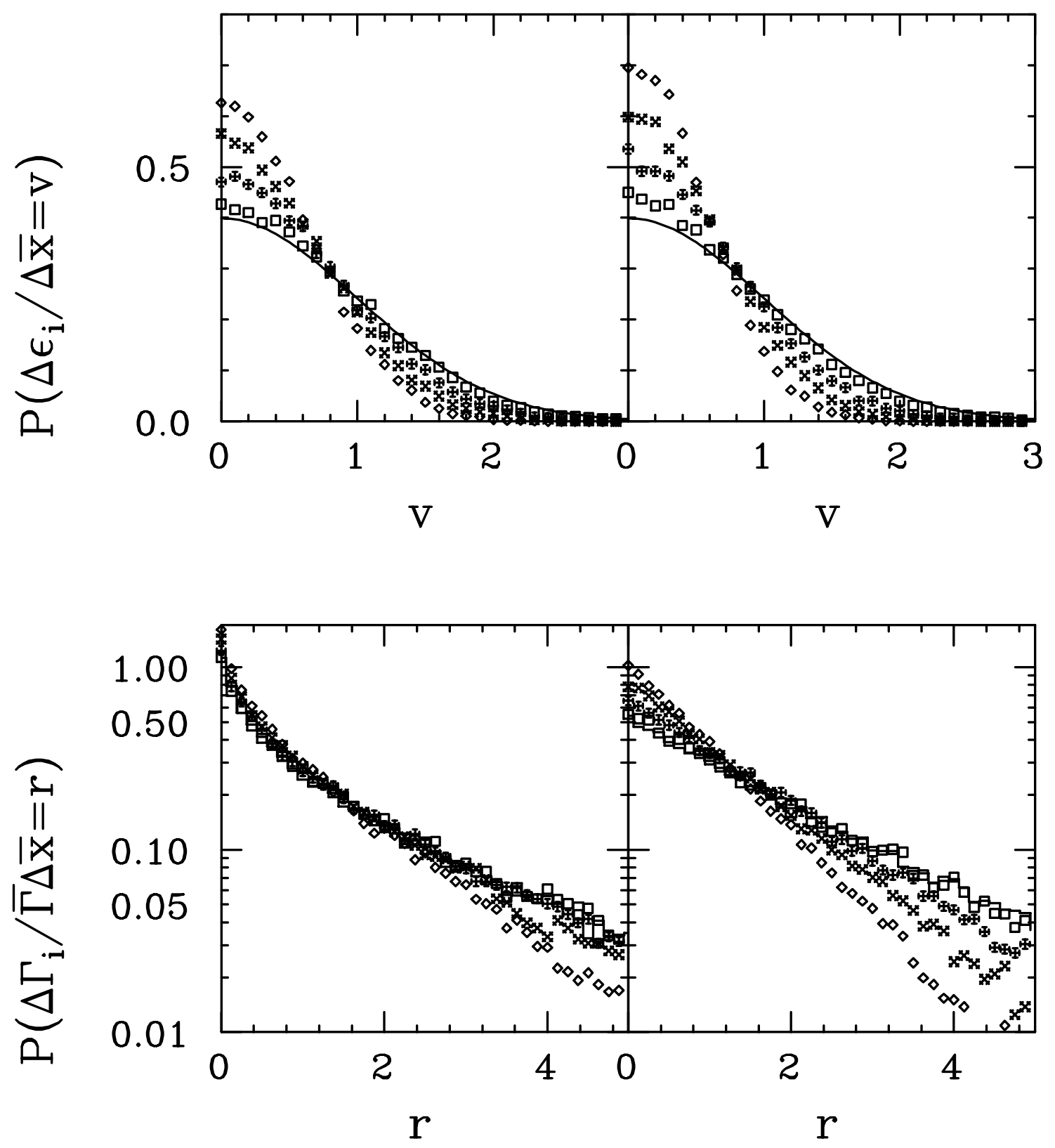


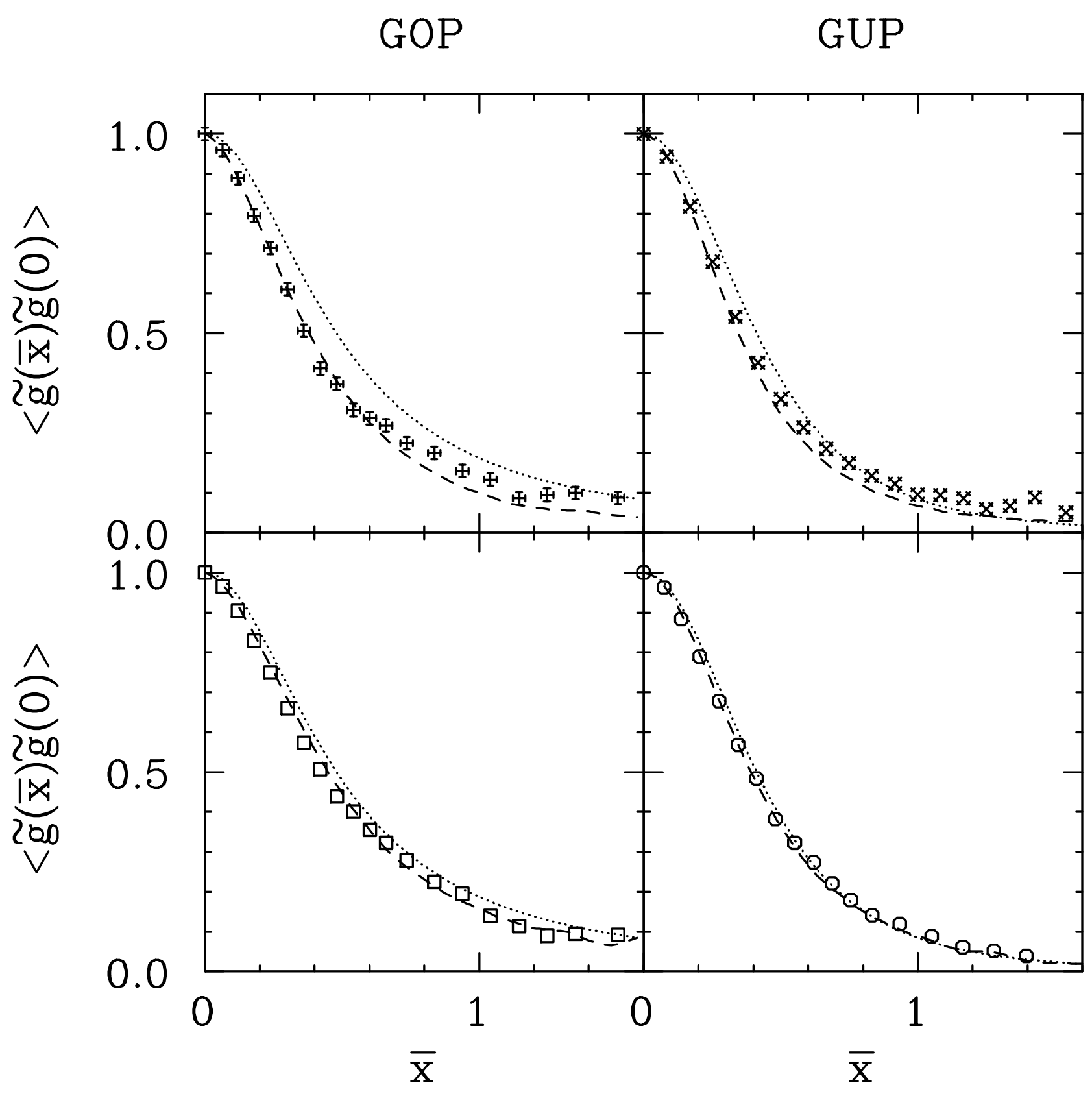

